This item was submitted to Loughborough's Research Repository by the author.

Items in Figshare are protected by copyright, with all rights reserved, unless otherwise indicated.

\title{
Microneedle-assisted microparticle delivery by gene guns: experiments and modeling on the effects of particle characteristics.
}

\section{PLEASE CITE THE PUBLISHED VERSION}

http://dx.doi.org/10.3109/10717544.2014.887158

\section{PUBLISHER}

(C) Informa Healthcare USA, Inc.

\section{VERSION}

AM (Accepted Manuscript)

\section{LICENCE}

CC BY-NC-ND 4.0

\section{REPOSITORY RECORD}

Zhang, Dongwei, Chris D. Rielly, and Diganta Bhusan Das. 2014. "Microneedle-assisted Microparticle Delivery by Gene Guns: Experiments and Modeling on the Effects of Particle Characteristics.". figshare. https://hdl.handle.net/2134/14354. 
This item was submitted to Loughborough's Institutional Repository (https://dspace.lboro.ac.uk/) by the author and is made available under the following Creative Commons Licence conditions.

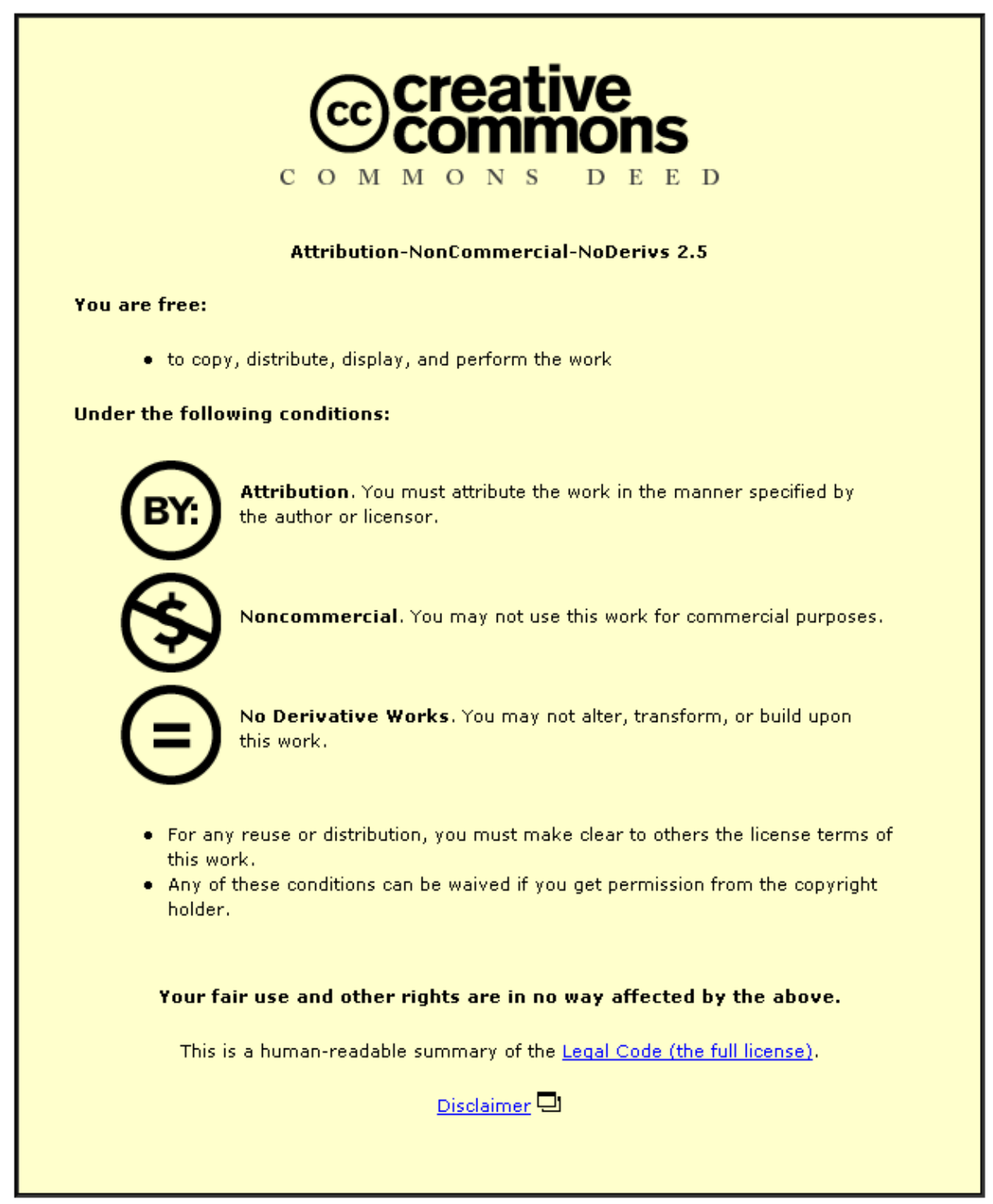

For the full text of this licence, please go to: http://creativecommons.org/licenses/by-nc-nd/2.5/ 
Microneedle Assisted Micro-particle Delivery by Gene Guns: Experiments and Modelling on the Effects of Particle Characteristics

Dongwei Zhang, Chris D Rielly, Diganta B Das*

Department of Chemical Engineering, Loughborough University, Loughborough LE113TU, UK

Short title: Microneedle Assisted Micro-particle Delivery

Revised version submitted for review and publication in

Drug Delivery

21 January 2014

*Corresponding author; Email: d.b.das@lboro.ac.uk 


\title{
Microneedle Assisted Micro-particle Delivery by Gene Guns: Experiments and Modelling on the Effects of Particle Characteristics
}

\author{
Dongwei Zhang, Chris D Rielly, Diganta B Das* \\ Department of Chemical Engineering, Loughborough University, Loughborough LE113TU, UK \\ (*Corresponding author; Email: d.b.das@lboro.ac.uk)
}

\begin{abstract}
Microneedles (MNs) have been shown to enhance the penetration depths of micro-particles delivered by gene gun. This paper aims to investigate the penetration of model micro-particle materials, namely, tungsten (<1 $<\mathrm{m}$ diameter) and stainless steel (18 and $30 \mu \mathrm{m}$ diameters) into a skin mimicking agarose gel to determine the effects of particle characteristics (mainly particle size). A number of experiments have been processed to analyse the passage percentage and the penetration depth of these micro-particles in relation to the operating pressures and MN lengths. A comparison between the stainless steel and tungsten micro-particles has been discussed, e.g. passage percentage, penetration depth. The passage percentage of tungsten micro-particles is found to be less than the stainless steel. It is worth mentioning that the tungsten micro-particles present unfavourable results which show that they cannot penetrate into the skin mimicking agarose gel without the help of MN due to insufficient momentum due to the smaller particle size. This condition does not occur for stainless steel micro-particles. In order to further understand the penetration of the micro-particles, a mathematical model has been built based on the experimental set up. The penetration depth of the micro-particles is analysed in relation to the size, operating pressure and MN length for conditions which cannot be obtained in the experiments. In addition, the penetration depth difference between stainless steel and tungsten micro-particles is studied using the developed model to further understand the effect of an increased particle density and size on the penetration depth.
\end{abstract}

Key words: Gene gun, microneedle, micro-particles, micro-particle density, micro-particle size, penetration depth, passage percentage

\section{Introduction}

Gene gun systems have been designed primarily as needle free techniques which can accelerate DNA loaded micro-particles to provide sufficient momentum so that they can breach the outer layer of the skin and achieve the purposes of gene transfection (Kis et al., 2012; Soliman, 2011; Walters et al., 2007; Kendall et al., 2004). Generally, the epidermis layer of the skin is considered as the main target of these micro-particles (Soliman et al., 2011; Liu, 2006; Quinlan et al., 2001; Bennett et al., 1999; Trainer et al., 1997). However, cell and tissue damages are particular problems for the use of gene guns (Obrien et al., 2011; Uchida et al., 2009; Thomas et al., 2001; Sato et al., 2000; Yoshida et al., 1997). In principle, reduction of the operation pressure in the gene guns (Xia et al., 2011; Uchida et al. 2009; Yoshida et al., 1997) and particle size (Obrien et al., 
2011) can minimize the cell/tissue damage but these tend to decrease the particle momentum and, hence, the penetration depths of the micro-particles in the tissue. In order to resolve these issues, a series of experiments which combine solid microneedles (MNs) with an in-house micro-particle delivery system (see Figure 1) has been reported recently by Zhang et al. $(2013 a, 2014)$. The potential of a system that combines microneedles and gene guns has been discussed in details by Zhang et al. (2013b).

MNs are minimally invasive microstructures that can pierce the outer layer of skin, namely the stratum corneum, almost painlessly which has been shown to enhance drug delivery rate (e.g., Olatunji and Das, 2011; Nayak et al., 2013; Olatunji et al., 2013; Donnelly et al., 2012). They are generally classified into 'solid' and 'hollow' microneedles (e.g., Al-Qallaf et al., 2009; Olatunji and Das, 2010; Olatunji et al., 2012; Nayak and Das, 2013; Han and Das, 2013; Zhang et al., 2013a,b). The solid MNs are able to penetrate the human skin to make holes (Kalluri et al., 2011; Davis et al., 2004; McAllister et al., 2003) as well as deliver drugs/genes which are coated (Cormier et al., 2004) or encapsulated (Miyano et al., 2005). The MN holes can also be used by the biolistic system for the delivery of the micro-particles. In this case, the micro-particles can penetrate with less resistance into the skin through the holes and further achieve an enhanced penetration depth to allow gene transfection in deeper tissue. Zhang et al. (2014) have used the experimental setup of $\mathrm{MN}$ assisted micro-particle delivery to fire biocompatible stainless steel micro-particles having an average diameter of $18 \mu \mathrm{m}$ into a skin mimicking agarose gel. Their results have shown that a number of micro-particles penetrate through the holes and achieve a considerable increase in the maximum penetration depth, e.g., a penetration depth of $1272 \pm 42 \mu \mathrm{m}$ was achieved inside the gel using $1500 \mu \mathrm{m}$ long MNs (AdminPatch MN 1500) as compared to the cases where the same gel and operating conditions were used and yet little or no penetration of MNs was observed due to the absence of MNs.

The overall process of MN assisted transdermal micro-particle delivery from gene guns consists of three main stages, which are the acceleration, separation and deceleration stages (Zhang et al., 2013a, 2014). These stages are identified in Figure 1. As can be seen, the experimental set up stores the pressurized gas in a gas receiver to a desired pressure which is detected by a digital pressure transducer. The release of the pressurized gas is operated by a solenoid valve. In addition, it is worth mentioning that the system uses a ground slide which can prevent the impact of pressurized gas on the target tissue and release the gas from the ventholes in the barrel. The acceleration stage is a major part of the particle delivery process as it is within this stage the micro-particles are accelerated to sufficient velocities using the pressurized gas as a driving source. To achieve these, the micro-particles are compressed and bound loosely into a pellet form and loaded into a ground slide in the system (Figure 1). As the ground slide is accelerated by the pressurised gas, the pellet containing the micro-particles is also accelerated. The separation stage is then followed which is aimed at separating the pellet into individual particles by a mesh although it is possible that this stage produces a few agglomerates. 
The deceleration stage involves two steps as follows: (i) the transport of the micro-particles in a space between mesh and target and (ii) the penetration of micro-particles into the chosen target. Previously, Zhang et al. (2014) used an experimental rig (Figure 1) to deliver stainless steel micro-particles into a skin mimicking agarose gel so as to demonstrate the feasibility of MN assisted micro-particle delivery. The results have shown that an enhanced penetration depth of micro-particles inside the agarose gel can be achieved using

MN assisted micro-particle delivery at a much lower injection pressure compared to typical gene guns.

Generally, the route of the micro-particle penetration in the target tissue is divided into two types which are the extracellular and intercellular routes (Zhang et al., 2013b; Soliman and Abdallah, 2011; Mitchell et al., 2003). The extracellular route may occur with large particles due to failures between the cell boundaries (Soliman, 2011). For example, Mitchell et al. (2003) have fired stainless steel micro-particles of $25 \mu \mathrm{m}$ average diameter into canine buccal mucosa using light gas gun (Crozier et al., 1957), which is governed by the micro-particle transport through extracellular route. However, the disadvantage of this is that large particle sizes may cause a lot of damage or destroy the skin surface.

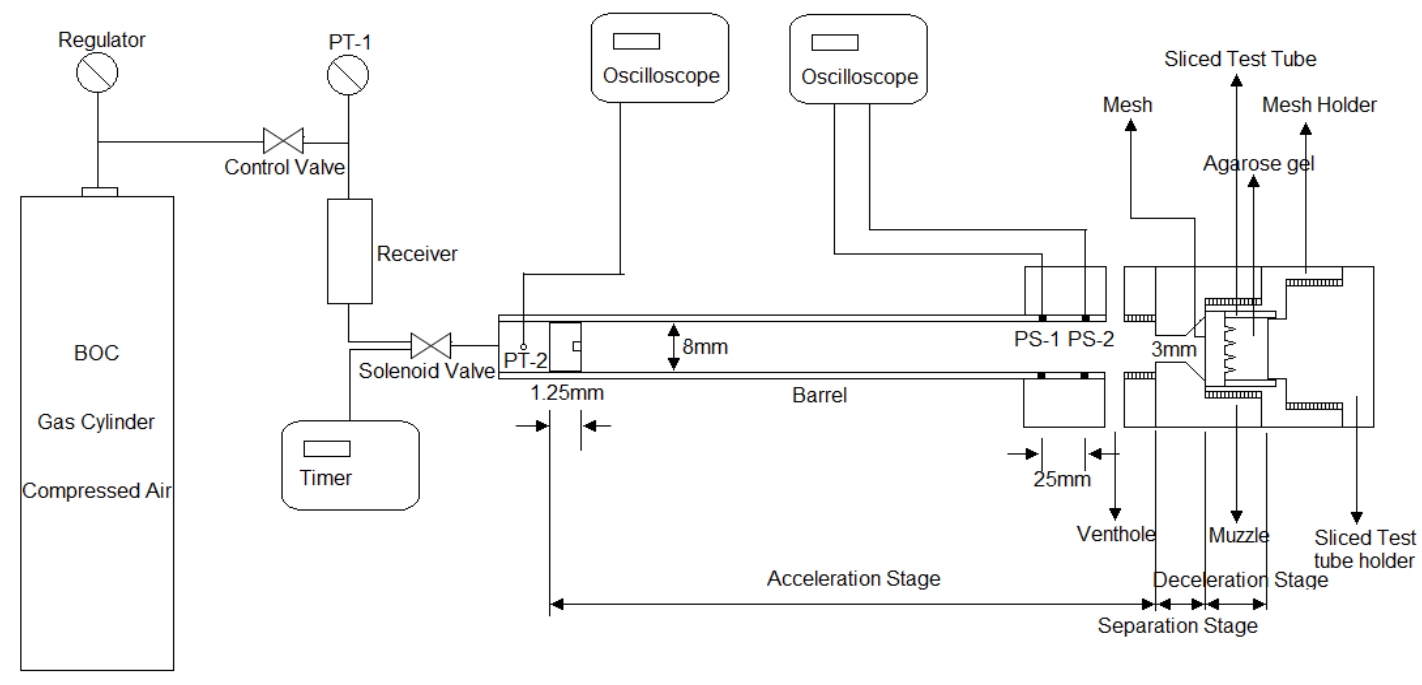

Figure 1: A schematic diagram of the experimental rig for the MN assisted micro-particle delivery system (Zhang et al., 2014)

The intercellular route is considered to be important in the case of delivering small particles, which pass through individual cell membranes, e.g., it can be used for particle mediated DNA immunization (Soliman and Abdallah, 2011; Hardy et al., 2005). Dense materials (e.g. gold and tungsten) are often made into small particles of a diameter ranging from 0.6 to $6 \mu \mathrm{m}$ (Soliman, 2011; Hardy et al., 2005) which are smaller than typical cell diameters. The particles can be accelerated to higher speeds to obtain enough momentum to breach the skin and penetrate through the individual cell membranes. Although gold is recommended for the particle delivery due to its high density, low toxicity and lack of chemical reactivity (Macklin, 2000), it is an expensive material. Several studies for the golden micro-particles delivery have been made, which show that 
the gold micro-particles can breach the human skin and penetrate to a depth ranging from 35 - $135 \mu \mathrm{m}$ at 30 bar operating pressure (Arora et al., 2008; Giudice et al., 2006). Kendall et al. (2004) have used a convergent-divergent device to accelerate gold particles of $1.8 \mu \mathrm{m}$ diameter which achieve a maximum penetration depth of $78.6 \mu \mathrm{m}$ in the skin at 60 bar pressure. Mitchell et al. (2003) have found that gold particle of 1 - $3 \mu \mathrm{m}$ diameter can reach a depth of around $60 \mu \mathrm{m}$ in canine tissue at an injection particle velocity of 550 $\pm 50 \mathrm{~m} / \mathrm{s}$. Recently, O'Brien et al. (2011) have reported that the penetration depth of golden micro-particles of $1 \mu \mathrm{m}$ diameter in mouse ear tissue is $50 \pm 11 \mu \mathrm{m}$ when the Helios gene gun is used at an operating pressure of 5.1 bar (75 psi).

In general, the intercellular route is the preferred penetration route due to less cell and tissue damages for the small micro-particles. In order to validate the effect of the MN assisted micro-particle delivery on the small micro-particles, tungsten micro-particles may be chosen to analyse the penetration in the skin mimicking agarose gel. Tungsten micro-particles are considered to be a good substitute for golden micro-particles for research of biolistic micro-particle delivery because of their cost effectiveness compared to gold particles, despite the fact they can be toxic to cells (Bastian et al, 2009; Yoshimisu et al, 2009; Russell et al, 1992). For example, Klein et al. (1987) first used a particle gun to accelerate spherical tungsten particles of $4 \mu \mathrm{m}$ diameter into epidermal cells of onions. Williams et al. (1991) have used a helium-driven apparatus to study $3.9 \mu \mathrm{m}$ average diameter tungsten and of golden particles, with a diameter ranged from 2 to $5 \mu \mathrm{m}$, on mouse liver. Recently, the review paper by Zhang et al. (2013b) has discussed the importance of particle characteristics and operation condition on the delivery of the micro-particles in detail.

Motivated by the above work, this paper aims to investigate the effects of particle characteristics on MN assisted micro-particle delivery by using micro-particles (e.g., tungsten micro-particles, biomedical grade stainless steel) of different densities and particle sizes. The study also gives an indirect comparison between the extracellular and intercellular routes using MN assisted micro-particle delivery. Zhang et al. (2013a) determined the passage percentage of stainless steel micro-particles at various conditions, e.g., mesh pore size, Polyvinylpyrrolidone (PVP - binder) concentration and operating pressure. They pointed out the required mesh pore size and PVP concentration of pellets for MN assisted micro-particle delivery for given particle size. This paper aims to revisit the same questions to determine the dependence of particle characteristics using tungsten and stainless steel micro-particles with the help of Zhang et al. (2014)'s experimental rig. A skin mimicking agarose gel is used as a target to determine the difference in penetration depth between tungsten and stainless steel micro-particles. In order to further understand the particle size and density effect on the penetration depth, a mathematical model of $\mathrm{MN}$ assisted micro-particle delivery is built using MATLAB (Version R2012b). The mathematical model is used to analyse the theoretical penetration depth of those two materials of micro-particles and further to verify the experimental results. In addition, the model aims to investigate the effect of a range of target properties (e.g. density, viscosity) on the maximum penetration 
depth of micro-particles. The developed model is also used to determine the characteristics of the micro-particle delivery where experimental data are not available. Please note that the paper is focused on modelling the micro-particle delivery process. The issues related to the loading of genes on these particles and subsequent gene transfection in a target cells are not discussed in this paper.

Please note that most of the technologies for loading DNA onto the particles and delivering the DNA-loaded particles are well developed. In the present context, we do not propose any changes to these existing methods but suggest a modification where the particle delivery process/devices are coupled with microneedles. The potential of these method are discussed in detail by Zhang et al. (2013a,b) and Zhang et al. (2014).

\section{Materials and experimental methods}

\subsection{Materials}

Irregular shaped tungsten micro-particles were purchased form Sigma-Aldrich Company Ltd (Gillingham, UK). It is considered as a good replacement for gold particles because its density is similar to gold and has a low cost (Menezes et al., 2012). Stainless steel mesh with pore size of $122 \mu \mathrm{m}$ was obtained from MeshUK, Streme Limited (Marlow, UK). Irregular shaped and spherical biocompatible stainless steel micro-particles were purchased from Goodfellow Cambridge Ltd. (Huntingdon, UK) and LPW Technology Ltd. (Daresbury, UK), respectively. Agarose powder (Sigma-Aldrich Company Ltd., Gillingham, UK) was used to prepare a skin mimicking agarose gel which was used a target for the micro-particles penetration experiments. Detailed method of preparation for this gel is given by Zhang et al. (2014).

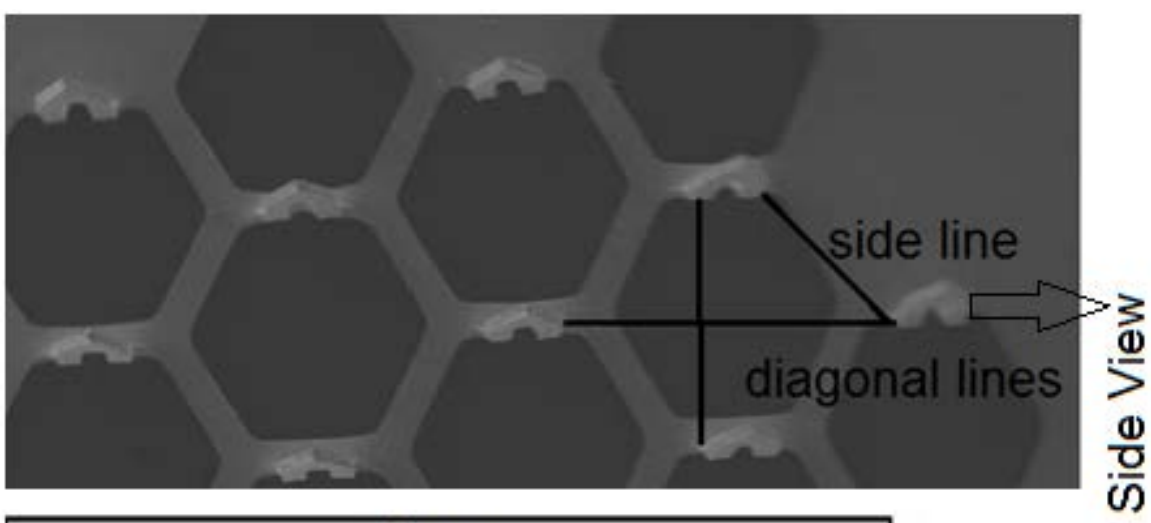

$6 \mathrm{~mm}$

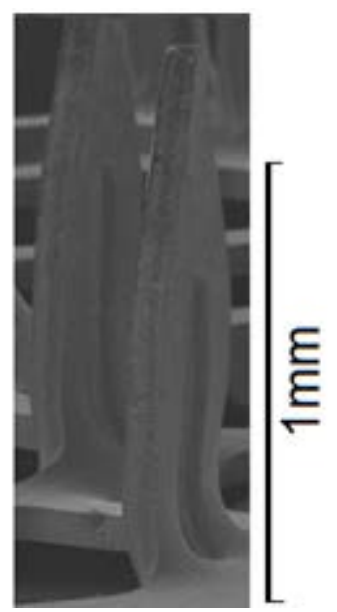

Figure 2: The image of AdminPatch 1500 (Note: MNs of AdminPatch 1200 are distributed as a diamond pattern) (Zhang et al., 2014)

Two different solid MN arrays which vary in the lengths of the MNs (AdminPatch 1200 and 1500) were purchased from nanoBioSciences limited liability company (Sunnyvale, CA, USA). The MNs are distributed as a diamond shape (see Figure 2) on a $1 \mathrm{~cm}^{2}$ circular area. In addition, an in-house fabricated MN array is 
used which consists of $750 \mu \mathrm{m}$ long MNs. The characteristic of each MN array is presented in Table 1. Although the results from the use of different MNs may be different, a range of dimensions of the MNs provide us the opportunity to understand the effects of a geometric parameter more accurately. Therefore, we consider it to be important to use more than one $\mathrm{MN}$ in our experiments despite differences in their geometries.

\subsection{Characterization of tungsten micro-particles}

The particle size distribution of tungsten powder is analysed by a particle size analyser (Mastersizer 2000, Malvern Instruments, Ltd, Malvern, Worcestershire, UK). It is found to range from 0.198 to $1.439 \mu \mathrm{m}$ with a mean diameter of $0.58 \mu \mathrm{m}$ and Sauter mean diameter of $0.49 \mu \mathrm{m}$. The particles can be viewed in the SEM image in Figure 3. The tungsten particles are highly agglomerated and have rough surfaces. From the SEM image, 50 random micro-particles are chosen to measure the average sphericity of the tungsten micro-particles which is found to be $0.66 \pm 0.13 \mu \mathrm{m}$. The average bulk density and porosity of tungsten powder are $7.43 \pm 0.08 \mathrm{~g} / \mathrm{cm}^{3}$ and $59.6 \pm 0.4 \%$. Ultimately, by combining the SEM image results with the size distribution, gained from particle size analyser it is possible to confirm that the tungsten particles are a nano-size powder.

Table 1: The characterizations of the MN array (Zhang et al., 2014)

\begin{tabular}{lll}
\hline MN Name & Parameters & Value $(\boldsymbol{\mu m})$ \\
\hline Adminpatch MN 1500 & Length & 1500 \\
& Width & 480 \\
& Thickness & 78 \\
& Space between MNs & 1546 \\
& Spaces on diagonal directions (see Figure 2) & 1643 \\
Adminpatch MN 1200 & & 3000 \\
& Length & 1200 \\
& Width & 480 \\
& Thickness & 78 \\
& Space between MNs & 1252 \\
& Spaces on diagonal directions & 1970 \\
& & 2426 \\
& Length & 750 \\
& Diameter & 250 \\
& Space between MNs & 500 \\
\hline
\end{tabular}

\subsection{Characterization of the pellet}

For the $\mathrm{MN}$ assisted micro-particle delivery, the micro-particles are required to be in the form a compressed cylindrical pellet which is prepared by a pellet press as described by Zhang et al. (2013a). Furthermore, the packing density should be homogeneous within the pellet as far as possible so as to increase the possibility that when the pellet is broken into particles and one obtains uniformly separated particles. This is an 
important characteristic of the developed process. Heterogeneity in the packing of the pellet is likely to create more uncertainty in the distribution of the separated particles. To prepare the cylindrical pellet, Zhang et al. (2013a) have mixed $40 \mathrm{mg}$ of PVP in $1 \mathrm{ml}$ of ethanol in which an amount of $0.035 \mathrm{~g}$ stainless steel micro-particles has been added and bind the particles together. The mixture is transferred to a pellet press (Zhang et al., 2013a) and compressed into a solid cylindrical pellet. Ethanol is evaporated due to their high volatility and therefore, it is not present in the dry pellet which is used in experiments. A similar method was followed to produce the pellets in this work. Furthermore, the homogeneity of the micro-particle packing was checked using a micro computed tomography (micro-CT). Micro-CT is an advanced non-destructive 3D imaging technique which can be used to clearly understand the internal microstructure of the samples (Ritman, 2004). Micro-CT has been used to detect any internal damage (e.g., internal fracture in the porous pellet) and/or heterogeneity in the packing of pellet in this work. As an example, a three dimensional view of the stainless steel pellet is shown in Figure 4a. As can be seen, the stainless steel micro-particles are compressed into a homogenous cylindrical pellet with approximately $2 \mathrm{~mm}$ in diameter and length. Although the top and button surfaces of the pellet are slightly uneven because the pellet gets stuck on the pellet press, it is not a big concern as it seems the packing density of the particles does not vary. The internal top view of the pellet at the position of $1.08 \mathrm{~mm}$ on the $\mathrm{z}$ axis is shown in Figure $4 \mathrm{~b}$ and the size view of the internal structure of the pellet at the position of $1.08 \mathrm{~mm}$ on the $y$-axis is shown in Figure 4c. It shows that PVP (white spots) is non-uniformly distributed in the pellet. PVP fills the void space in the micro-particles causing them to bind loosely together as indicated by the white circles (PVP) in the Figure 4b-c. The above figures cannot clearly show the PVP combination of the pellet. Therefore an SEM view is provided which show the top surface of a pellet (see Figure 5 a).

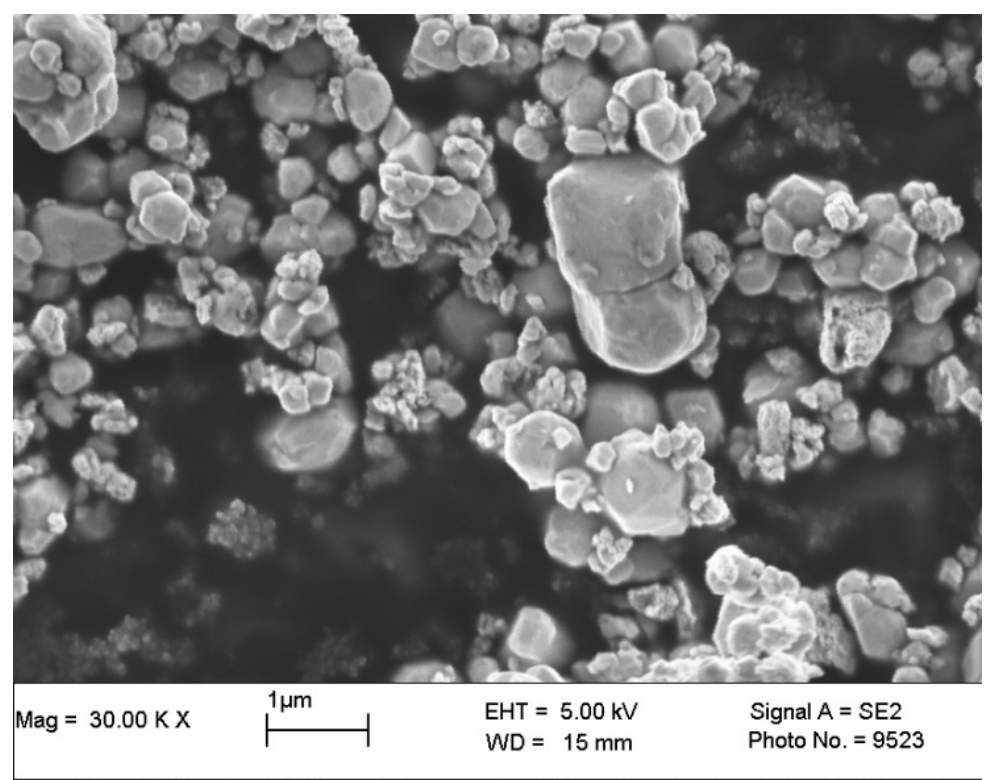




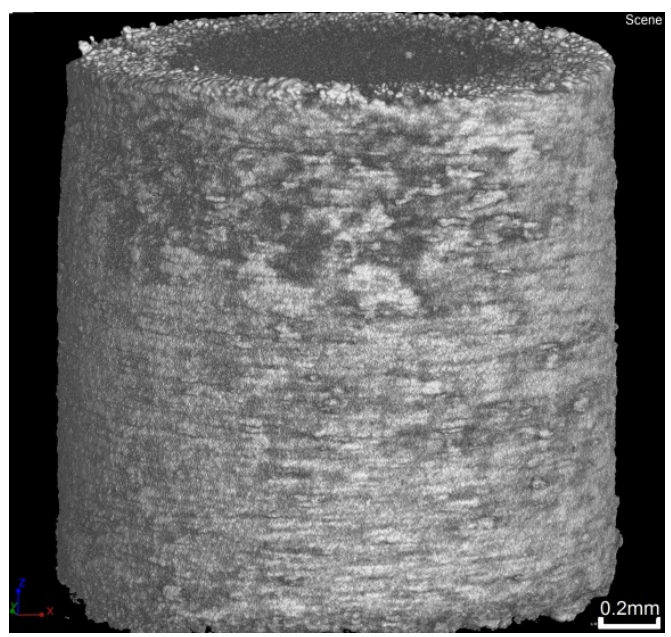

(a)

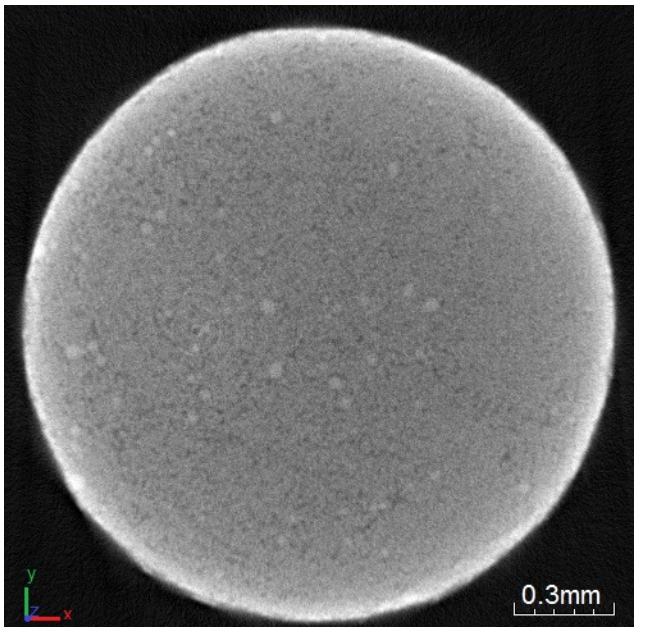

(b)

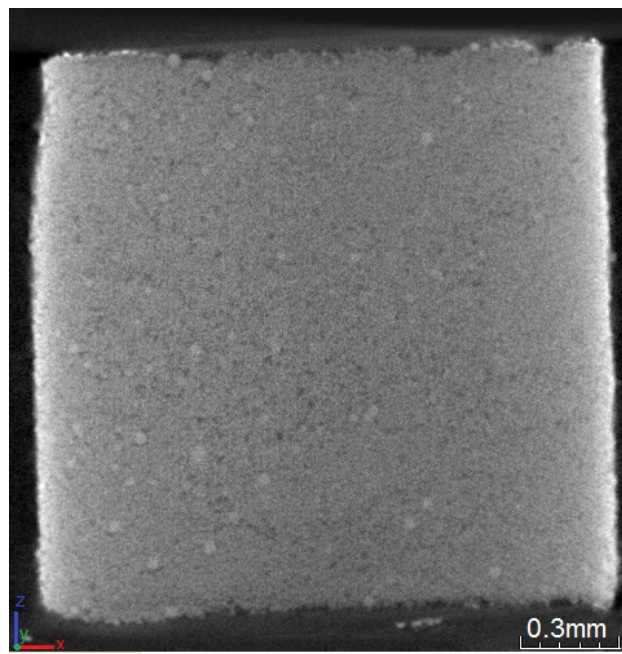

(c)

Figure 4: Micro-CT images of a stainless steel micro-particle pellet made of $40 \mathrm{mg} / \mathrm{ml} \mathrm{PVP}$ (white spots in the images): (a) reconstructed three dimension view of the pellet (b) top internal view across the pellet at the position of $1.08 \mathrm{~mm}$ on the $z$ axis (c) side internal view across the pellet at the position of $1.08 \mathrm{~mm}$ on the $y$ axis. The images show homogeneity of the packing of the micro-particles.

Figure 5(a) clearly shows the characterization of the pellet top surface. As can be seen, the micro-particles are bound to form a uniform pellet surface. As expected, the PVP is distributed non-uniformly around the pellet, which agrees with results detected by the micro-CT (Figure 4). Thus, it may cause the pellet to separate into a few agglomerates after the separation stage due to increased bind strength between some of micro-particles. Previously, Zhang et al. (2013a) have analysed the passage percentage of the pellet and size distribution of the separated particles using various operating pressure and different binder (PVP) concentration. They show that $40 \mathrm{mg} / \mathrm{ml}$ PVP in a pellet is ideal as it almost separates the pellet into individual micro-particles with some small agglomerates using $178 \mu \mathrm{m}$ pore size of mesh. As presented in the Figure $5 b$, the application of mesh pore size presents a good control of the size distribution of the separated micro-particles and uses to the following experiments for the investigation of the penetration depth of 

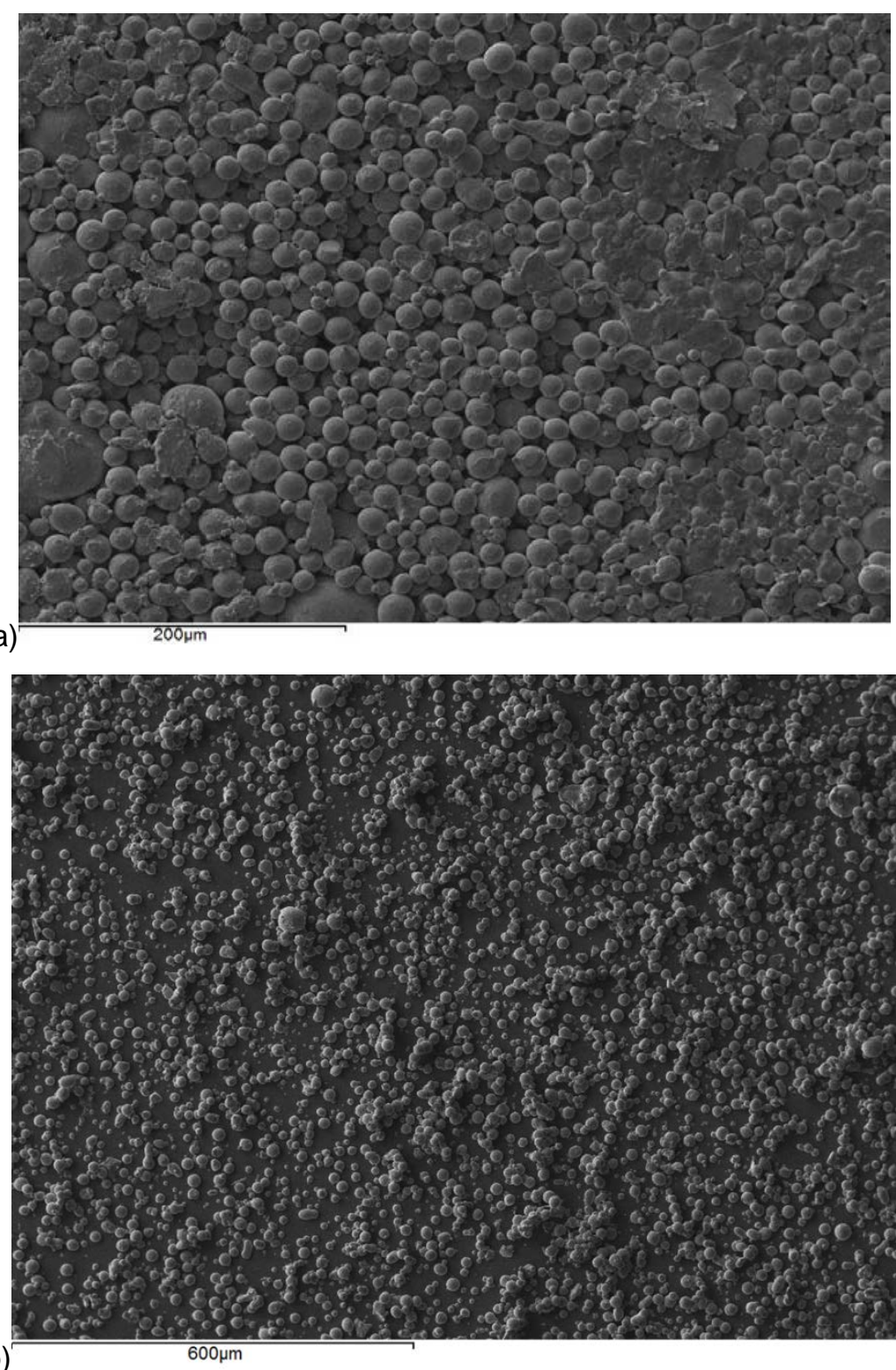

Figure 5: (a) SEM image of the top surface of the pellet (b) SEM image of the separated micro-particles from a pellet which is made of $40 \mathrm{mg} / \mathrm{ml}$ PVP concentration and fired at a pressure of 20 bar and separated using a mesh of $178 \mu \mathrm{m}$ pore size

\subsection{Modelling of micro-needle assisted micro-particle delivery: governing equations}

The detailed theoretical principle of each stage is discussed in the following sections.

\subsubsection{Acceleration stage}

253 For the MN assisted micro-particle delivery, a pellet is attached to a ground slide which is accelerated by a 254 pressurized gas in the acceleration stage (see Figure 1). It indicates that only the pressurized gas has done the work during the acceleration process. The equation to describe this process: 


$$
E=\int_{0}^{L} P_{2} \pi R^{2} d l
$$

Where $E$ is the kinetic energy of the pellet, $L$ is the traveling distance of the ground slide in the barrel before the pellet is broken into micro-particles and $\mathrm{R}$ is the radius of the barrel. The gas pressure to push ground slide moving forward is $P_{2}$ which decreases from a pressure $P_{1}$ in front of the barrel. The gas expansion can be described by the Boyle's law (Webster, 1995) as below:

$$
\mathrm{P}_{1} \mathrm{~V}_{1}^{\gamma}=\mathrm{P}_{2}\left(\mathrm{~V}_{1}+\mathrm{V}_{2}\right)^{\gamma}
$$

Where $\gamma$ is the heat capacity ratio which defines as a constant of 1.6 for monatomic gas or 1.4 for diatomic gas.

The kinetic energy of the pellet attached ground slide at the end of the barrel can also be calculated as:

$$
\mathrm{E}=\frac{1}{2} \mathrm{Mu}^{2}
$$

Where $\mathrm{M}$ is the mass of the pellet attached ground slide and $\mathrm{u}$ is the velocity of the ground slide.

From an energy conservation point of view, the ground slide velocity can be specified as follows:

$$
\mathrm{u}=\sqrt{\frac{2 \mathrm{P}_{1} \mathrm{~V}_{1}^{\gamma}\left[\left(\mathrm{V}_{1}+\pi \mathrm{R}^{2} \mathrm{~L}\right)^{1-\gamma}-\left(\mathrm{V}_{1}\right)^{1-\gamma}\right]}{\mathrm{M}(1-\gamma)}}
$$

\subsubsection{Separation stage}

In theory, the pellet is released from the ground slide at end of the barrel and separated by a mesh in the separation stage. Let us assume that the pellet has a mass of $m_{p}$ and it is separated into $n$ micro-particles with the same mass $\mathrm{m}$ and they have the same velocity $\mathrm{u}_{1}$ after passing through the mesh. Further, the energy loss is assumed to be $\mathrm{x}$ in the process of the pellet separation. According to the energy conservation law to describe the kinetic energy of the separated micro-particles which is given as:

$$
\frac{1}{2} \mathrm{nmu}_{1}^{2}=(1-\mathrm{x}) \times \frac{1}{2} \mathrm{~m}_{\mathrm{p}} \mathrm{u}^{2}
$$

The equation (5) can be rearranged to an equation which uses to calculate the velocity of the separated micro-particle and gives as:

$$
\mathrm{u}_{1}=\sqrt{1-\mathrm{x}} \times \mathrm{u}
$$

\subsubsection{Deceleration stage}

The deceleration stage of the micro-particles is an important stage which directly affects the penetration depth of micro-particle in the skin. The drag force of the skin is the major factor to decelerate the micro-particles. In this work, the particle penetration in the skin use Newton's second law to proportional the change rate of particle momentum to the drag force $f_{d}$ :

$$
\mathrm{f}_{\mathrm{d}}=-\mathrm{m} \frac{\mathrm{du} \mathrm{d}_{\mathrm{d}}}{\mathrm{dt}}
$$


Various studies have adopted that the drag force acting on the micro-particles is split into a yield force ( $\left.F_{y}\right)$, a frictional resistive force $\left(F_{f}\right)$ and a resistive inertial force of target material $\left(F_{i}\right)$ (Soliman et al., 2011; Liu, 2007; Mitchell et al., 2003; Kendall et al. 2001; Dehn 1987). Thus, equation (7) is written as:

$$
-\left(F_{i}+F_{f}+F_{y}\right)=m \frac{d u_{d}}{d t}
$$

The equations for each resistant force are shown as below:

$$
\mathrm{F}_{\mathrm{i}}=6 \pi \mu_{\mathrm{t}} \mathrm{r}_{\mathrm{p}} \mathrm{u}_{4}
$$

$$
\mathrm{F}_{\mathrm{f}}=\frac{1}{2} \rho_{\mathrm{t}} \mathrm{A}_{\mathrm{c}} \mathrm{u}_{4}^{2}
$$

$$
\mathrm{F}_{\mathrm{y}}=3 \mathrm{~A}_{\mathrm{c}} \sigma_{\mathrm{y}}
$$

Where $\mu_{t}$ is the viscosity of the target, $r_{p}$ is the radius of the micro-particle, $\rho_{t}$ is the density of the target and $\sigma_{y}$ is the yield stress of the target.

\subsection{Modelling strategy and parameters}

For the purpose of this paper, we use the software MATrix LABoratory (Matlab) to build and solve the theoretical model for MN assisted micro-particle delivery. The model consists of three main parts including a main program, an event program and two function programs (acceleration and deceleration stage). The main program simulates the whole process of the MN assisted micro-particle delivery. The event program defines the impact points of the micro-particles on the skin, rebound points on the boundary of the gap between mesh and skin and the end points inside the skin. The function programs input the equations, which are used to determine the theoretical results. The function programs are implemented to the main program by choosing a suitable ode solver which requires considering the condition of the function program (stiff/non-stiff). In addition, an if statement has been used to define the position of the micro-particles and further to confirm the selection of the equations to calculate the theoretical velocity of micro-particles at different position. A for statement has been used to repeat the simulation of a number of micro-particles in the deceleration stage.

In the model, human skin is considered as a target for micro-particles in the model. The three main layers of human skin, stratum cornum (SC), viable epidermis (VE) and dermis layers are considered in the model. The detailed skin properties are listed in the Table 2. It is worth to mention that the yield stress and density of the dermis layer is considered the same with the viable epidermis layer. Zhang et al. (2014) have used porcine skin instead of human skin to analyse the viscosity by using a rotational viscometer with parallel plate 
geometry. The result will be used in this study, which is shown in Table 2. The viscosity of each skin layer is treated as the same in the model.

Table 2: Skin properties used in the model

\begin{tabular}{lll}
\hline Parameter & Value & Reference \\
\hline Thickness of viable epidermis $(\mathrm{VE}), \mathrm{T}_{\mathrm{ve}}(\mathrm{m})$ & 0.0001 & Holbrook et al. (1974) \\
Thickness of stratum cornum(SC), $\mathrm{T}_{\mathrm{sc}}(\mathrm{m})$ & 0.00002 & $\begin{array}{l}\text { Matteucci et al. (2008); } \\
\text { Schaefer et al. (1996) }\end{array}$ \\
Yield stress of SC, $\mathrm{Y}_{\mathrm{sc}}(\mathrm{MPa})$ & & Wildnauer et al. (1971) \\
Density of $\mathrm{SC}, \rho_{\mathrm{sc}}\left(\mathrm{g} / \mathrm{cm}^{3}\right)$ & $3.2-22.5$ & Duck (1990) \\
Density of $\mathrm{VE}, \rho_{\mathrm{ve}}\left(\mathrm{g} / \mathrm{cm}^{3}\right)$ & 1.5 & Duck (1990) \\
Yield stress of $\mathrm{VE}, \mathrm{Y}_{\mathrm{ve}}(\mathrm{MPa})$ & 1.15 & Kishino et al. (1988) \\
Viscosity of skin, $\mu_{\mathrm{t}}(\mathrm{Pa} . \mathrm{s})$ & 2.2 & Zhang et al. (2014) \\
\hline
\end{tabular}

322

The lengths of the pierced holes are obtained from measuring the hole lengths in the skin mimicking agarose gel when MNs are inserted. In this case, this model is chosen to analyse the delivery of tungsten micro-particles. Zhang et al. (2014) have indicated that the pellet is separated into individual particles with a few agglomerates using a mesh and then penetrates into the target. It illustrates that a number of the tungsten particles agglomerated after the separation stage in the experiment. In order to correlate the model with the realistic experiments, a number of tungsten micro-particles are considered to the diameter of tungsten particle are considered to agglomerate together to be $3 \mu \mathrm{m}$ diameter after the separation stage in the model. Previously, Zhang et al. $(2013 a, 2014)$ have analysed the sauter diameter of the spherical and irregular stainless steel using a particle size analyser of Coulter. They also measured the average length of pierced holes in the skin mimicking agarose gel using a digital optical microscope. The length is considered uniformly and applied to the model in this case. The detailed set up of the particles properties and other relevant constants used in the model are listed in Table 3.

\section{Results and Discussions}

The purpose of this section is compare tungsten micro-particle with stainless steel micro-particles for the MN assisted micro-particle delivery based on the analysis of the passage percentage and the penetration depth inside target. The maximum penetration depth of micro-particles is analysed in relation to the operating pressure (see section 3.2.1) and MN length (see section 3.2.2). In addition, a theoretical model is used to analyse the penetration depth in relation to above two parameters to compare tungsten particle with stainless steel micro-particle and then to further understand the particle density effect on micro-particle penetration. 
Table 3: Relevant constants used in the developed model

\begin{tabular}{|c|c|}
\hline Parameter & Value \\
\hline Mass of ground slide with the pellet, M (g) & 1.25 \\
\hline Length of barrel, $L(m)$ & 0.5 \\
\hline Radius of barrel/ground slide, $R(m)$ & 0.00375 \\
\hline Volume of receiver, $V_{1}(L)$ & 1 \\
\hline Space between mesh and skin, $L_{1}(m)$ & 0.05 \\
\hline Density of tungsten $\left(\mathrm{g} / \mathrm{cm}^{3}\right)$ & 19.25 \\
\hline Density of stainless steel $\left(\mathrm{g} / \mathrm{cm}^{3}\right)$ & 8 \\
\hline Average Diameter of tungsten particle $(\mu \mathrm{m})$ & 3 \\
\hline Average Diameter of spherical stainless steel particle $(\mu \mathrm{m})$ & 18 \\
\hline Average Diameter of Irregular stainless steel particle $(\mu \mathrm{m})$ & 30 \\
\hline Viscosity of air, $\mu$ (Pa.s) & 1.78 \\
\hline \multicolumn{2}{|l|}{ Length of pierced holes $L_{p}(\mu m)$ : } \\
\hline Adminpatch 1500 & 1149 \\
\hline Adminpatch 1200 & 1048 \\
\hline In-house fabricated MN & 656 \\
\hline \multicolumn{2}{|l|}{ Width of pierced holes $L_{w}(\mu m)$ : } \\
\hline Adminpatch 1500 & 302 \\
\hline Adminpatch 1200 & 302 \\
\hline In-house fabricated MN & 156 \\
\hline
\end{tabular}

\subsection{Analysis of passage percentage}

In a previous study, Zhang et al. (2013a) have shown that stainless steel micro-particles yield a higher passage percentage and a good quality size distribution of separated micro-particles if $40 \mathrm{mg} / \mathrm{ml} \mathrm{PVP}$ (binder) concentration and a mesh with pore size of $178 \mu \mathrm{m}$ are used. Here, the pellets of tungsten micro-particles are operated at the same conditions as the previous study of the stainless steel micro-particles and to make a comparison of the passage percentage between those two micro-particles. The passage percentage is analysed in relation to the operating pressure which is varied from 2.4 to 4.5 bar. As shown in Figure 6 , the passage percentage is increased due to an increase in the operating pressures for each micro-particle. This is because the pellets gain more momentum at higher operating pressures, which in turn also causes the separated particles to gain more momentum while passing through the mesh. Zhang et al. (2013a) have indicated that the passage percentage of stainless steel micro-particles reaches a maximum due to some particles sticking to the mesh and some rebounding, hence not passing into the test tube (particle collector). As expected, the tungsten micro-particles show a similar performance to stainless steel. Figure 6 shows the passage percentage of tungsten micro-particles is less significant than stainless steel micro-particles. It might 

mesh and target except the agglomerates.

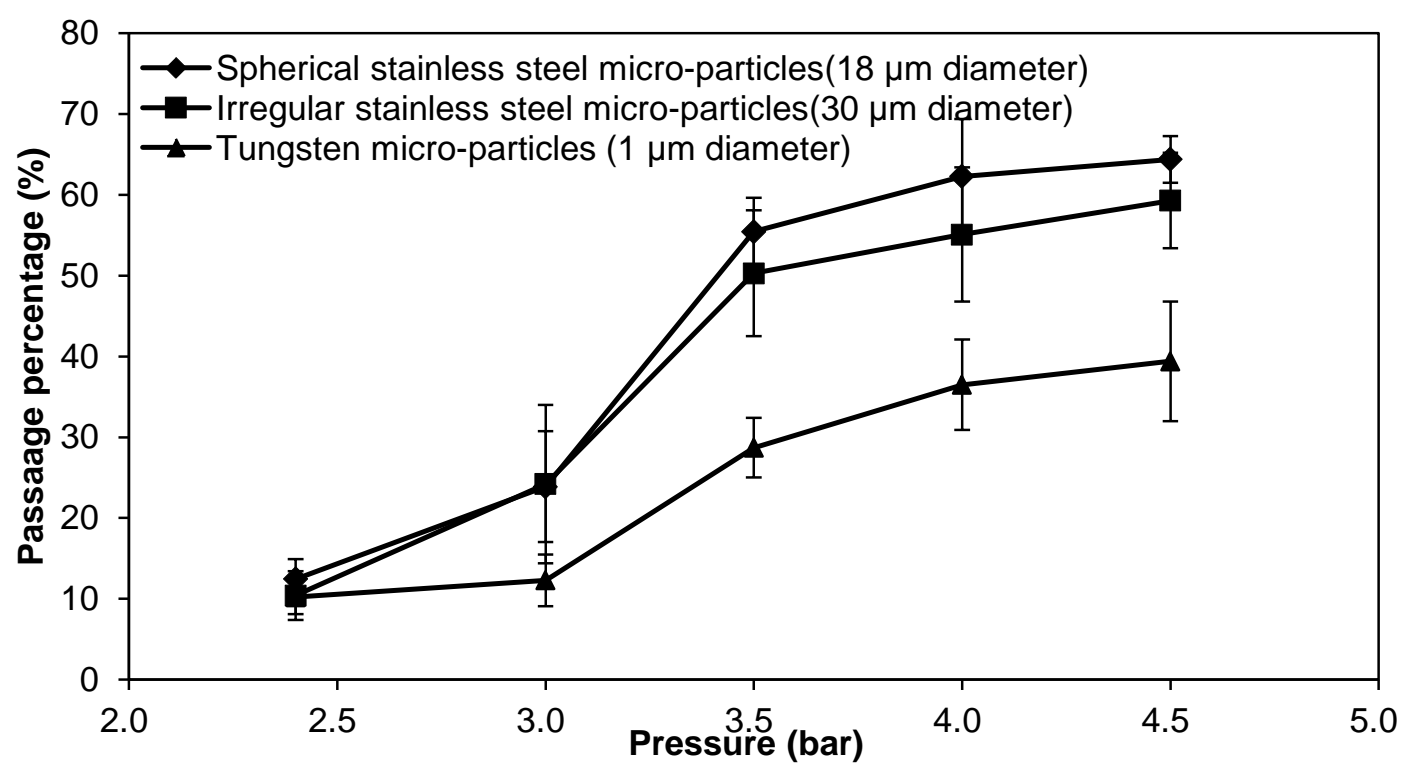

362

Figure 6: The effect of operating pressure on the Passage percentage of the pellet separation. Each curve in the figure is generated from three repeats of experiments (mesh pore size: $178 \mu \mathrm{m}$, PVP concentration: 40 $\mathrm{mg} / \mathrm{ml})$

\subsection{Experimental analysis of the penetration depth of micro-particles}

\subsubsection{Effect of the operating pressure}

The operating pressure is shown to be a key variable on the penetration depth of stainless steel micro-particles of 18 and $30 \mu \mathrm{m}$ average diameters by us in a previous study (Zhang et al., 2013a, 2014). In this paper, we aim to investigate the difference of the penetration depth between the larger stainless steel micro-particle and smaller tungsten micro-particles at various pressures. The tungsten micro-particles cannot penetrate into the skin mimicking concentration of agarose gel. This is because the momentum of the particles is insufficient to breach the surface of the gel. In the experiment, the operating pressures are kept between 3 to 5 bar which are low for the small particles to achieve velocity to breach the target. It requires a higher pressure for the small particles to achieve sufficient momentum to penetrate further into the target. In addition, a comparison between tungsten and stainless steel micro-particles is shown in Figure 7. As can be seen, both irregular and spherical stainless steel particles of 30 and $18 \mu \mathrm{m}$ average diameters achieve good penetration depths inside the skin mimicking agarose gel. Although the density of the stainless steel is lower than tungsten, larger diameters of stainless steel micro-particles increase their masses which lead to increased momentums of the micro-particles so as to allow them to penetrate further in the target (skin mimicking agarose gel). The two micro-particles show that the penetration depths increase due to an increase in the particle size. 
To further understand the effect of the particle density and size on the penetration depth in the target, the theoretical model is used to analyse the penetration of above two materials of micro-particles in a same target, which is discussed in the section 3.3.2. When the MNs are inserted into the skin, it creates holes which remain there after the removal of the MNs. The micro-particles can be delivered through these holes, thus compensating for the insufficient momentum. As presented in Figure 7 the penetration depths of the tungsten particles are greater inside the agarose gel when Adminpatch MN 1500 is used. This is because a number of particles can penetrate through the pierced holes. However, the maximum penetration depths of the micro-particles vary while the pressure increases from 3 to 5 bar. Zhang et al. (2014) have indicated that the length of the pierced holes is unable to maintain constantly, which varies the maximum penetration depth of the micro-particles inside the target. Thus, the variation in the length of the pierced holes directly affects the penetration depth of the micro-particles. It means that the holes length is a major factor to maximize the penetration depth in the MN assisted micro-particle delivery. The MN length effect on the micro-particle penetration depth is discussed in the section 3.2.2 in detail. Figure 7 also shows that the penetration depths of those two stainless steel micro-particles are more than the tungsten micro-particles. It indicates that an increased momentum due to increase in particle size/operating pressure of micro-particles affect the penetration depth in the MN assisted micro-particle delivery.

\subsubsection{Effect of the micro-needle length}

Zhang et al. (2014) have used three different lengths of MNs to study the maximum penetration depth differences of stainless steel micro-particles of 18 and $30 \mu \mathrm{m}$ diameters in a skin mimicking agarose gel. The characterizations of those three MNs are presented in Table 1. To further determine the effect of MN length on the penetration depth of small and dense micro-particles, those three MNs are used in this case. Figures $8 a-b$ show the penetration of the tungsten micro-particles in the skin mimicking agarose gel based on the assistance of Adminptach MN 1500 and the in-house fabricated MN 750, respectively. As can be seen, the maximum penetration depth of micro-particles after using Adminptach MN 1500 is more than in-house fabricated MN 750, but the number of micro-particles entering the pierced holes is less obvious. This is because a long needle increases the length of pierced holes in the agarose gel, which maximizes the penetration depth of micro-particles. In addition, the diameter of the in-house fabricated MN is greater than the thickness of the Adminptach MN 1500 MN (see, Table 1), which creates wider holes to provide the convenience for particle penetration. It indicates that a desired penetration depth and amount of micro-particles can be controlled by changing the MN length and width for further research. 


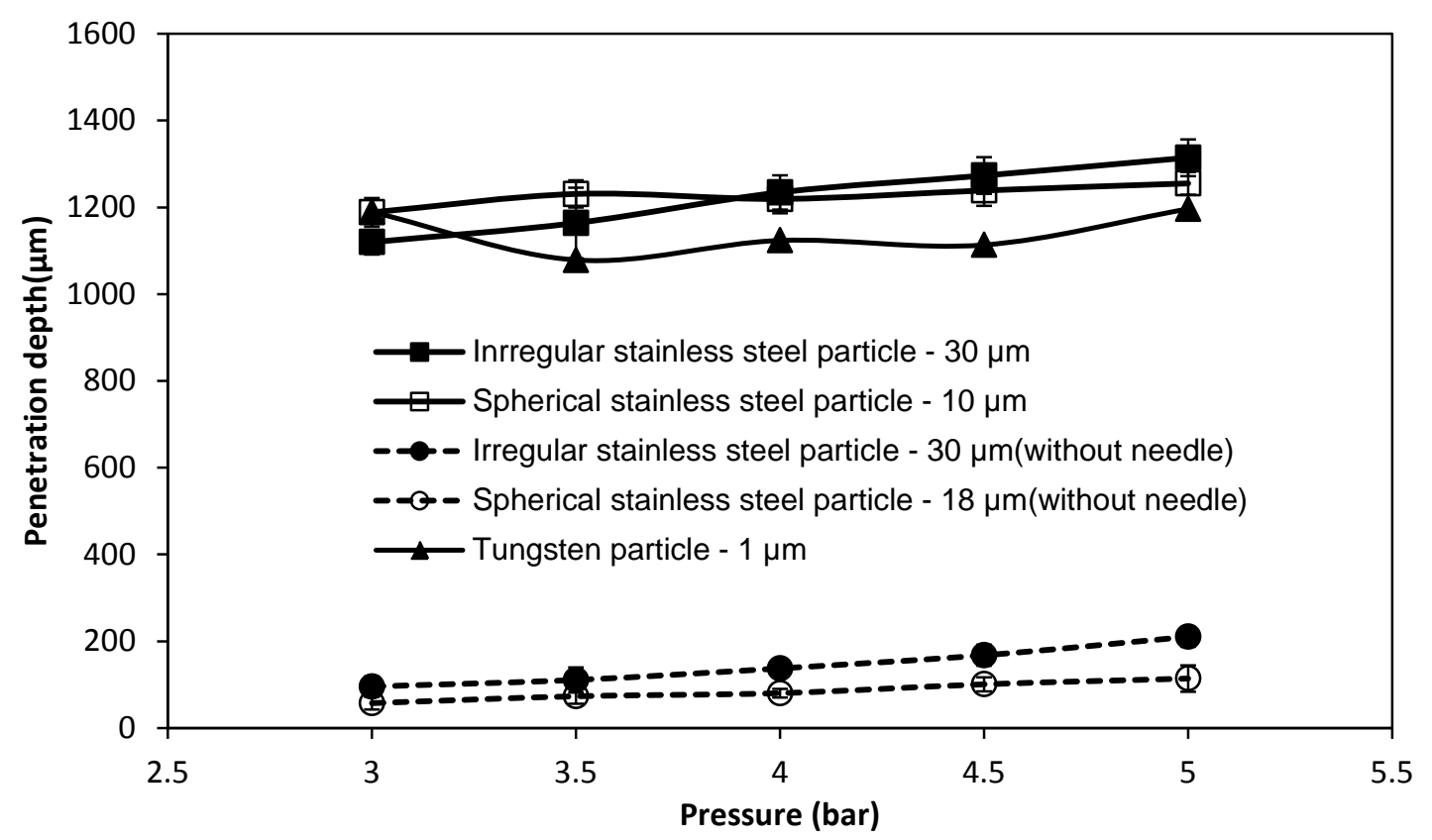

Figure 7: The effect of the operating pressure on the penetration depth of different type of particles (mesh:

$418178 \mu \mathrm{m}$ of pore size: dash line: particle penetration without using MN; solid line: particle penetration with MN).

419 Each curve in the figure is generated from three repeats of experiments.

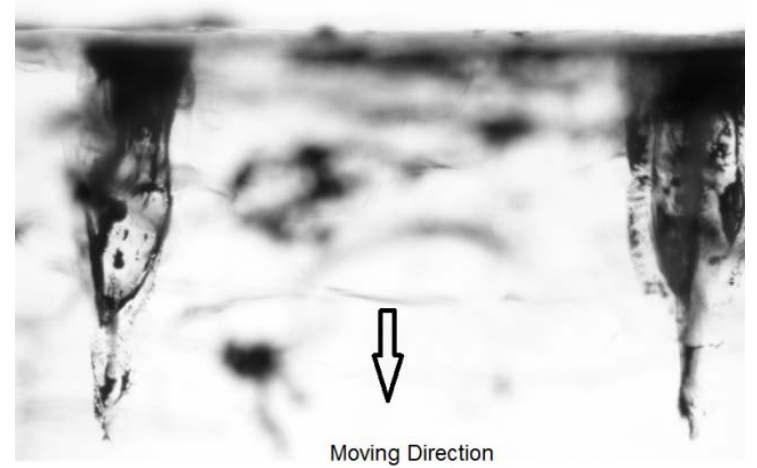

Moving Direction

(a)

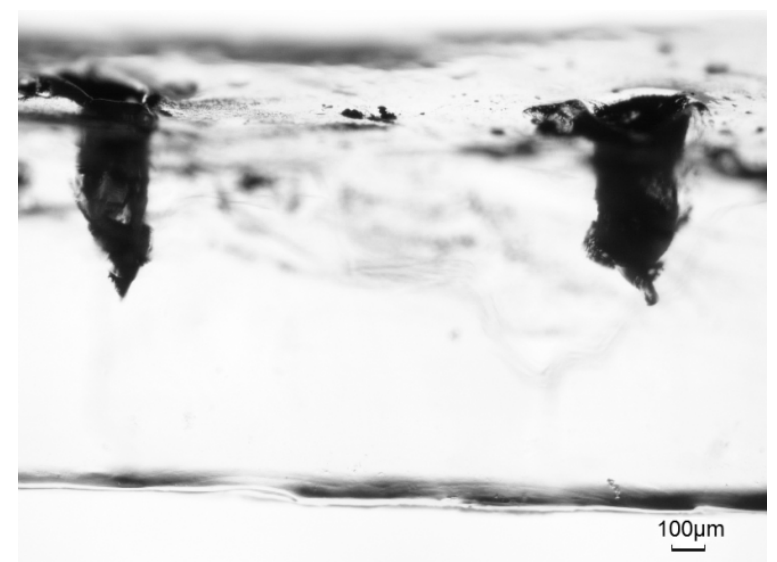

(b)

Figure 8: The penetration of tungsten micro-particles in the skin mimicking agarose gel based on the assistance of MNs (a) Adminpatch MN 1500 (b) In-house fabricated MN 750 


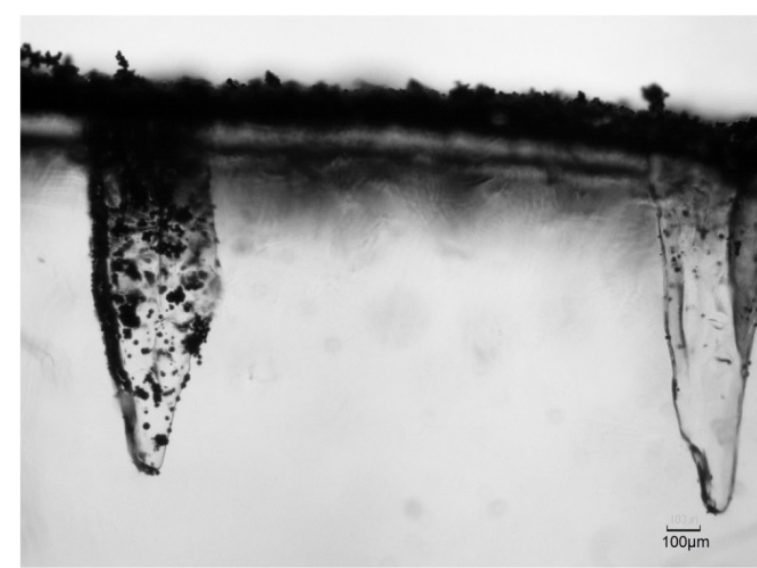

(a)

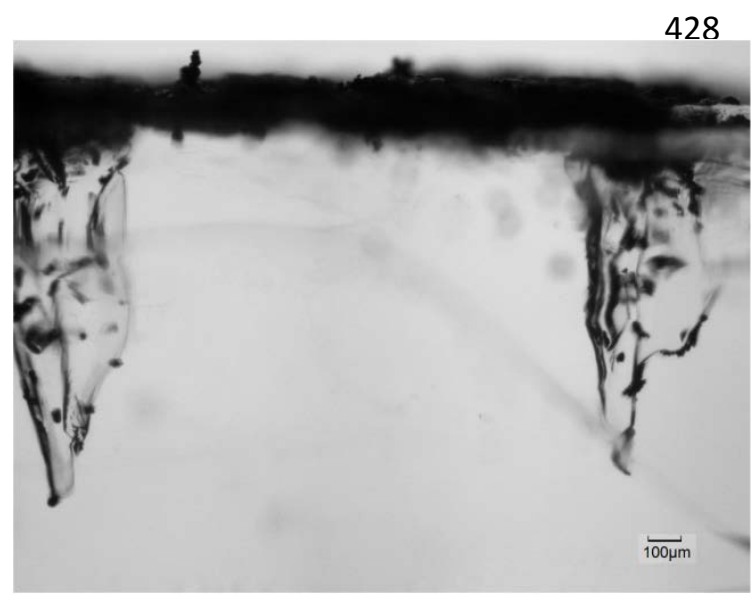

(b)

Figure 9: The penetration of stainless steel micro-particles in the skin mimicking agarose gel based on the assistance of MNs (a): spherical micro-particles of $18 \mu \mathrm{m}$ average diameters (b): irregular micro-particles of $30 \mu \mathrm{m}$ average diameters

Zhang et al. (2014) have fired spherical and irregular stainless steel micro-particles in the skin mimicking agarose gel. As presented in Figure 9, a number of micro-particles penetrated further through the pierced holes. It presents the same effect with the tungsten micro-particles. However, the amount of the stainless steel micro-particles that penetrates into the pierced holes is less than tungsten micro-particles if compared with the result in Figure 8. The number of irregular stainless steel micro-particles of $30 \mu \mathrm{m}$ average diameter is less than spherical micro-particle (18 $\mu \mathrm{m}$ diameter) in Figure 9. It indicates that the amount of micro-particle in the pierced holes decreases from an increase in particle size. This is because of the hole is reformed after the removal of the $\mathrm{MN}$, reduced the opening area of the hole on the top gel surface and further to affect the micro-particle penetration. Zhang et al. (2014) have shown that the thickness of the hole is only $78 \mu \mathrm{m}$ after the removal of Adminpatch MN 1500. It limits the amount of the micro-particles to penetrate into the holes, especially to the large size of irregular stainless steel micro-particles. However, the maximum penetration depth of each micro-particle is close but related to length of the pierced holes.

The maximum penetration depths of tungsten micro-particles show significant differences between each MN array as shown in Figure 10. It increases from an increase in MN length. As expected, a longer MN increases the length of the pieced holes and thereby increases the maximum penetration depth of micro-particles. However, the maximum penetration depth is varied at different operating pressure. This is because the length of the pierced holes is varied after the removal of the MN array. The above results show the advantage of MN assisted micro-particle delivery which provides a positive effect on the particle penetration even if the momentum of the particle is insufficient to breach the target. 


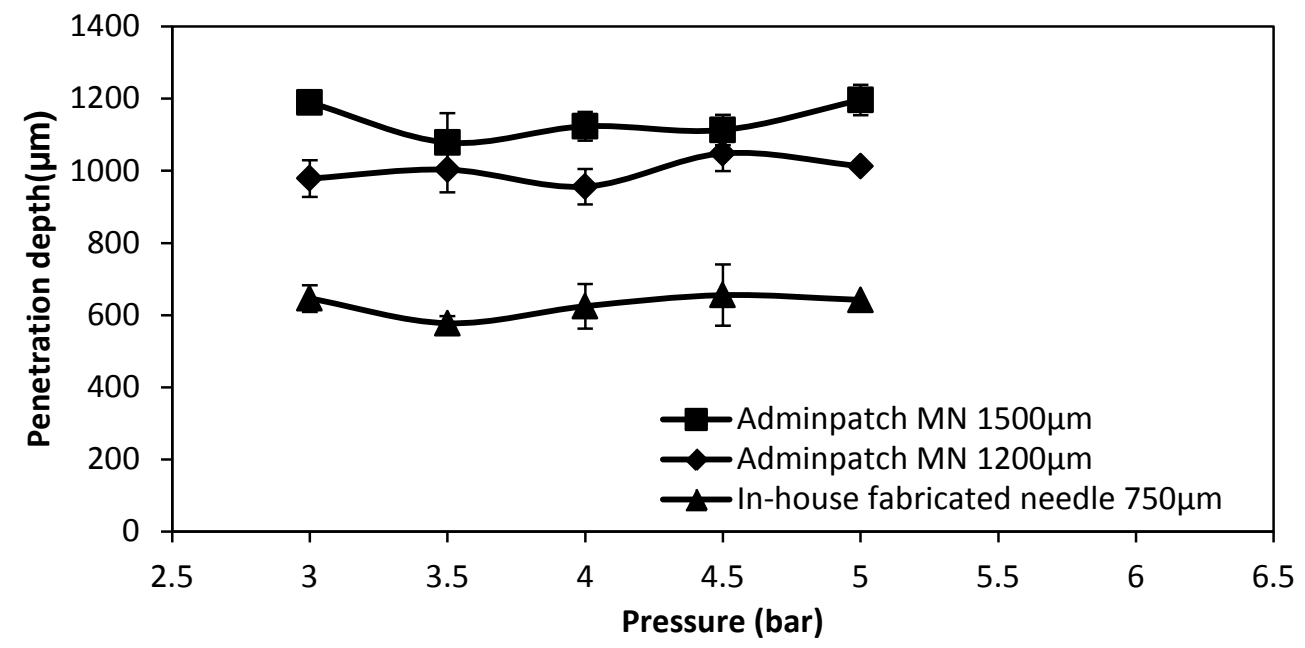

Figure 10: The maximum penetration depth of tungsten micro-particles in the skin mimicking based on the assistance of MNs (mesh pore size: $178 \mu \mathrm{m}$; each curve in the figure is generated from three repeats of experiments).

\subsection{Modelling the penetration of micro-particle in skin}

Firstly, this section aims to understand the micro-needle assisted micro-particle delivery based on the modelling of the trajectory of tungsten micro-particles in the deceleration stage. It is worth to mention that the tungsten micro-particle and stainless steel micro-particle may vary in shape and size which may affect results in reality. However, the both the stainless steel and tungsten micro-particles are assumed to be spherical with uniform size (average diameter) in the model. The maximum penetration depth is analysed in relation to the operating pressure, micro-needle length, particle size and density to further understand the difference between tungsten and stainless steel micro-particles for the MN assisted micro-particle delivery. Finally, a further analysis of the effect of each resistive force on the maximum penetration depth of micro-particles is discussed to verify the main factor which minimizes the penetration depth.

\subsubsection{Modelling the delivery of tungsten micro-particles}

Figure 11a shows the trajectories of increased diameter of tungsten micro-particles in the deceleration stage. The initial positions of the micro-particles are randomly selected at the beginning to mimic the separation of the pellet of tungsten micro-particles. In this case, we assumed that a number of tungsten micro-particles are stuck together where the micro-particles are spherical in shape and each micro-particle has diameter of $3 \mu \mathrm{m}$ after the separation stage (mesh). The trajectory is considered to be linear. The velocity decrease is represented by the colour change of the trajectory which corresponds to the colour bar in the figure. The velocity decreases slowly from approximately 135 to $110 \mathrm{~m} / \mathrm{s}$ from the mesh to the target. Figure 11a shows that a number of the micro-particles achieve further penetration depths via the pierced holes. The figure presents the similar performance of the developed model with experimental results. The detailed penetration process of tungsten micro-particles in the skin are shown in Figure 11b-c. Figure 11b shows the penetration 
489

490

491

492

493

494

495

496

497

498

499

of tungsten micro-particles in the top skin layer of stratum corneum at 5 bar operating pressure. As can be seen, the particle velocity deceases very fast in the stratum corneum due to an increased resistance; the penetration depth is approximately $0.7 \mu \mathrm{m}$ which could be ignored. Some particles are delivered through the pierced holes and then penetrate into the epidermis/dermis layer of the skin (side surface of the pierced holes), as shown in Figure 11c. It shows that the variation of the velocity is changed slightly in the pieced holes and decreased fast after penetrating the skin. It shows a similar performance as the penetration in the stratum corneum due to an increased resistance. Based on the above figures the penetration depth of tungsten micro-particles is negligible in the skin, which matches well with the experimental results.

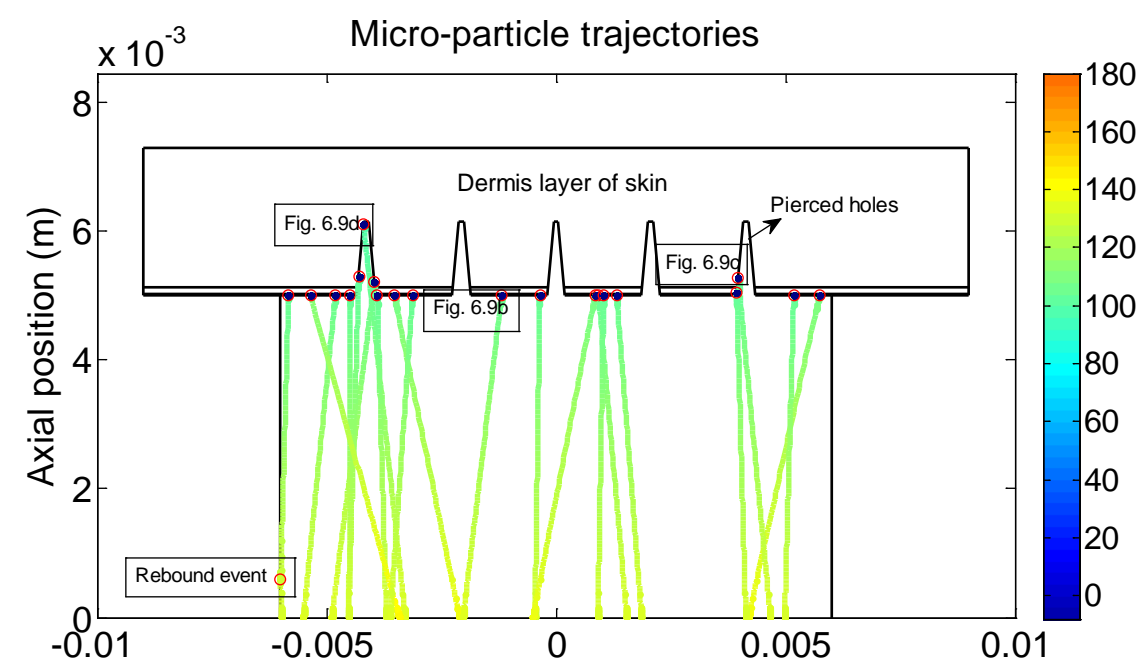

(a)

Radial position (m) 180 60 20 00 60 $\times 10^{-3} \quad$ Micro-particle trajectories

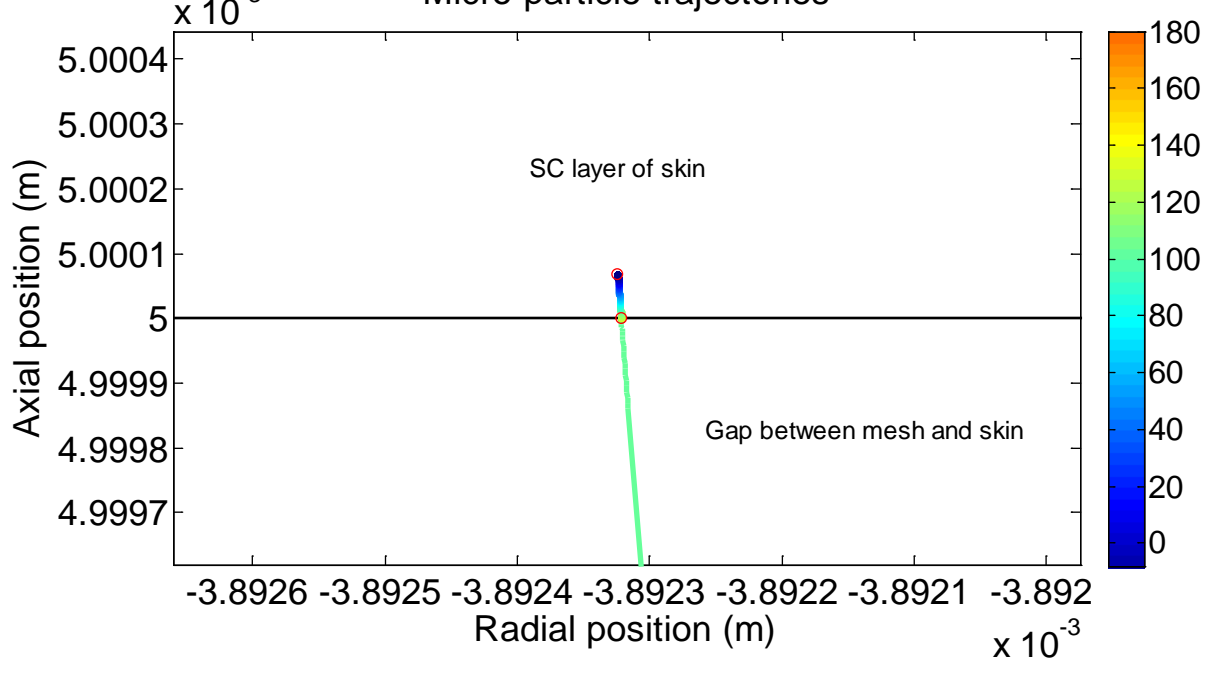



(c)

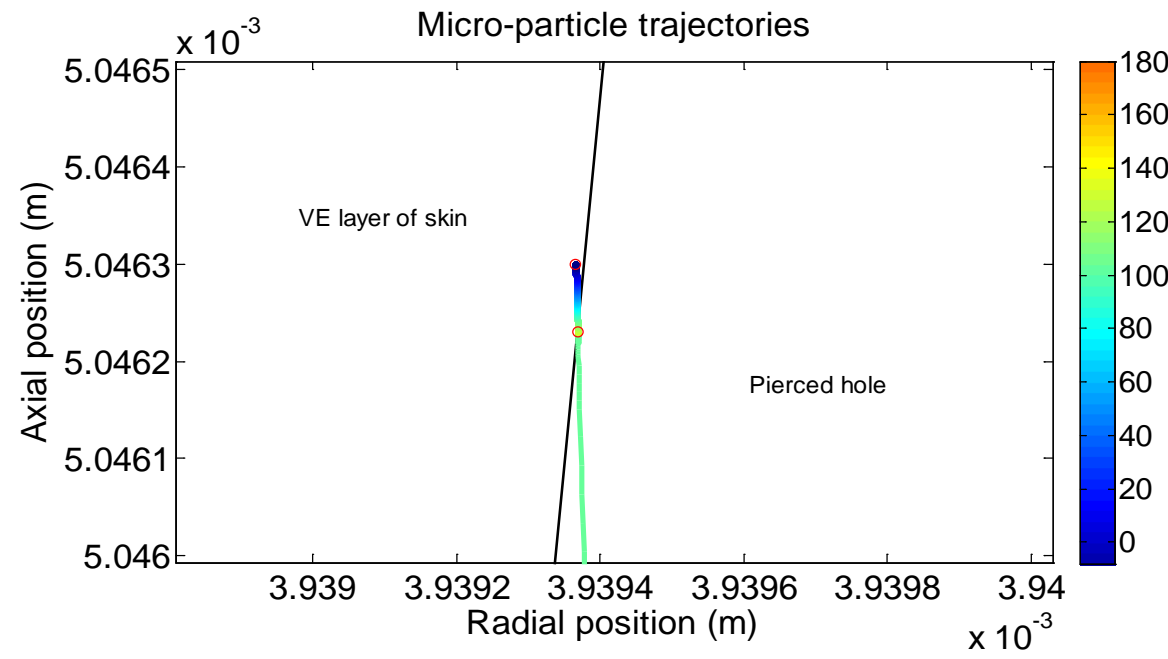

501 Figure 11: The trajectories of the tungsten micro-particles in the deceleration stage: (a). The overall view of

the micro-particle trajectories (b).The particle penetration at the area without needle hole (c).The view of the micro-particle penetrate through the needle hole (pressure: 5 bar)

\subsubsection{The effect of the operating pressure and particle size on the penetration depth}

In the experimental results, the operating pressure only presents a slight effect on the penetration depth of the tungsten micro-particle without using $\mathrm{MN}$. The pressures are varied from 3 to 5 bar, which are too low for small micro-particles when compared with previous gene gun research (Arora et al., 2008; Giudice et al., 2006; Kendal et al., 2004; Mitchell et al., 2003). In order to further understand the effect of the operating pressures on the penetration depth of this tungsten micro-particle, the penetration depth is analysed at various operating pressures which range from 3 to 60 bar in the model. As presented in Figure 12 the penetration depth of the tungsten particle is increased from 0.04 to $0.28 \mu \mathrm{m}$ without using MNs while the pressure varies from 3 to 60 bar. The penetration depth is negligible. It illustrates that the tungsten particle of $3 \mu \mathrm{m}$ diameter are too small for penetration even if the operating pressure increases to a great value. Figure 12 also shows the tungsten particle achieves a great penetration depth after using MNs. However, the penetration depth is increased slightly from an increase in operating pressure. 


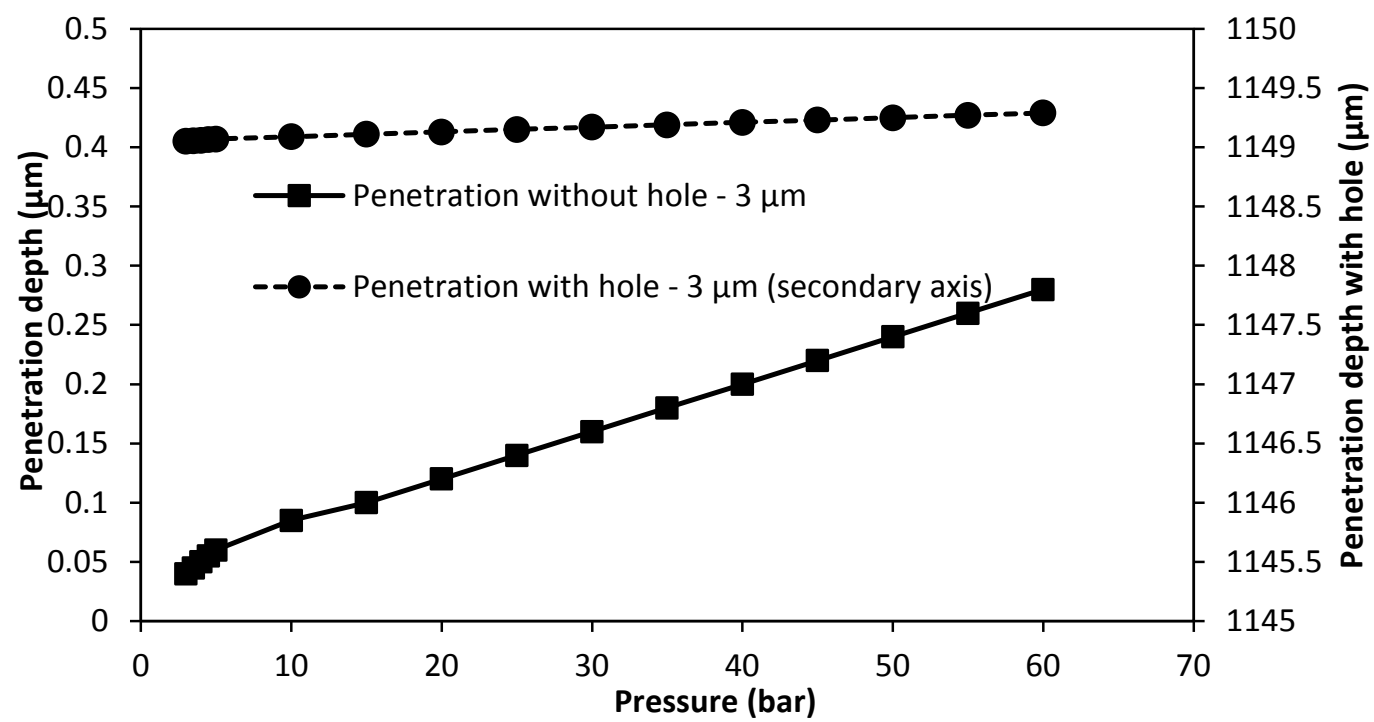

Figure 12: The effect of the operating pressure on the penetration of tungsten particle

To further understand the effects of particle size and density on the penetration depth, the particle is assumed to be spherical in shape with uniform size and the particle diameter is varied from 3 to $100 \mu \mathrm{m}$ for both stainless steel and tungsten micro-particles using the presented model. As presented in Figure 13 the penetration depth of tungsten micro-particles increases from 0.07 to $65.61 \mu \mathrm{m}$ in the skin without using MNs and from 1149.07 to $1214.61 \mu \mathrm{m}$ using Adminpatch MN 1500 at 5 bar pressure. It shows more penetration depth than stainless steel which only penetrates 0.02 to $17.36 \mu \mathrm{m}$ in the skin and 1149.02 to $1170.5 \mu \mathrm{m}$ using a same MN as tungsten while the particle diameter ranges from 3 to $100 \mu \mathrm{m}$. This comparison directly shows the advantage of dense particles on the penetration in the target for gene gun systems.

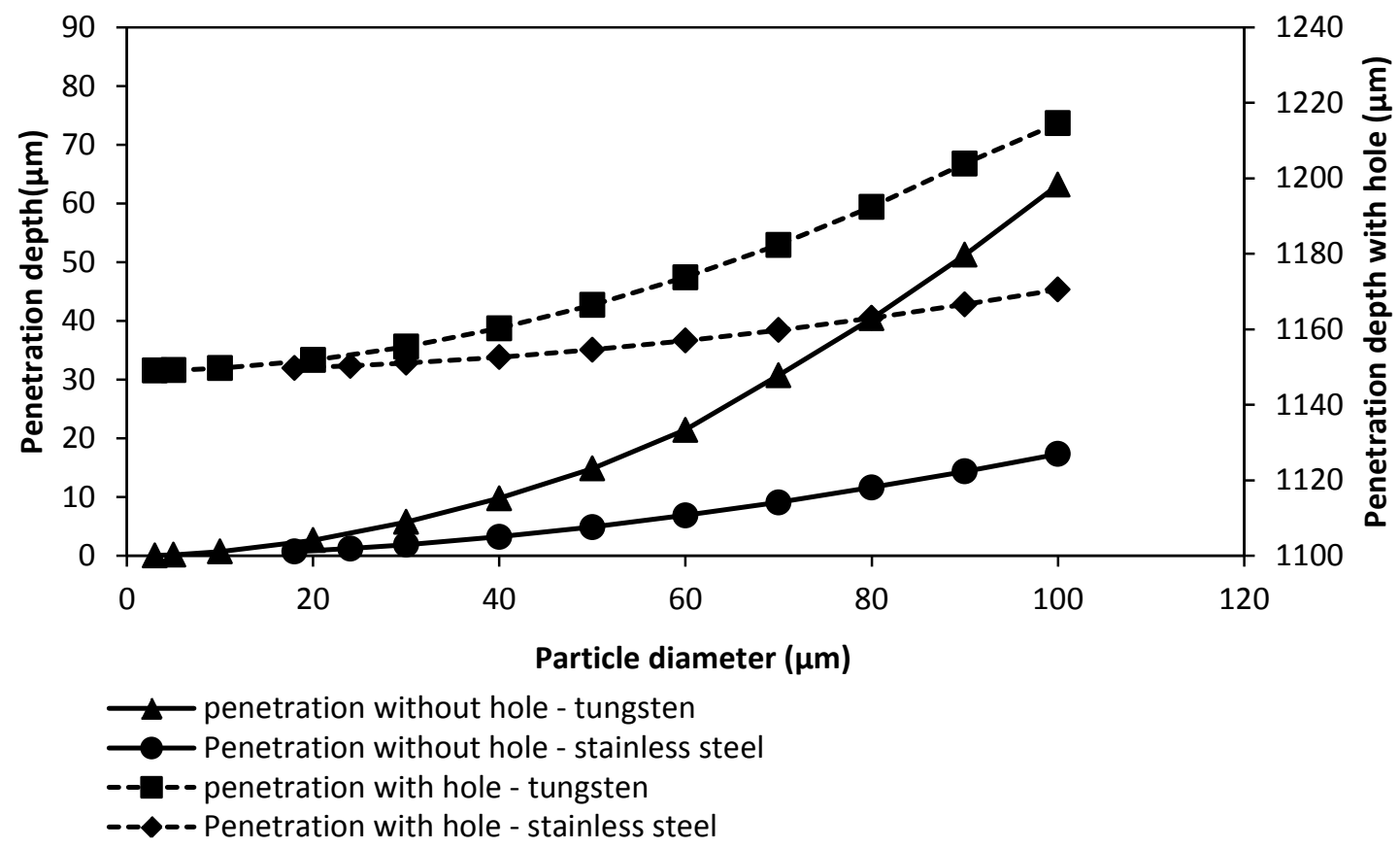

Figure 13: The effect of the tungsten particle size on the penetration depth (operating pressure: 5 bar) 


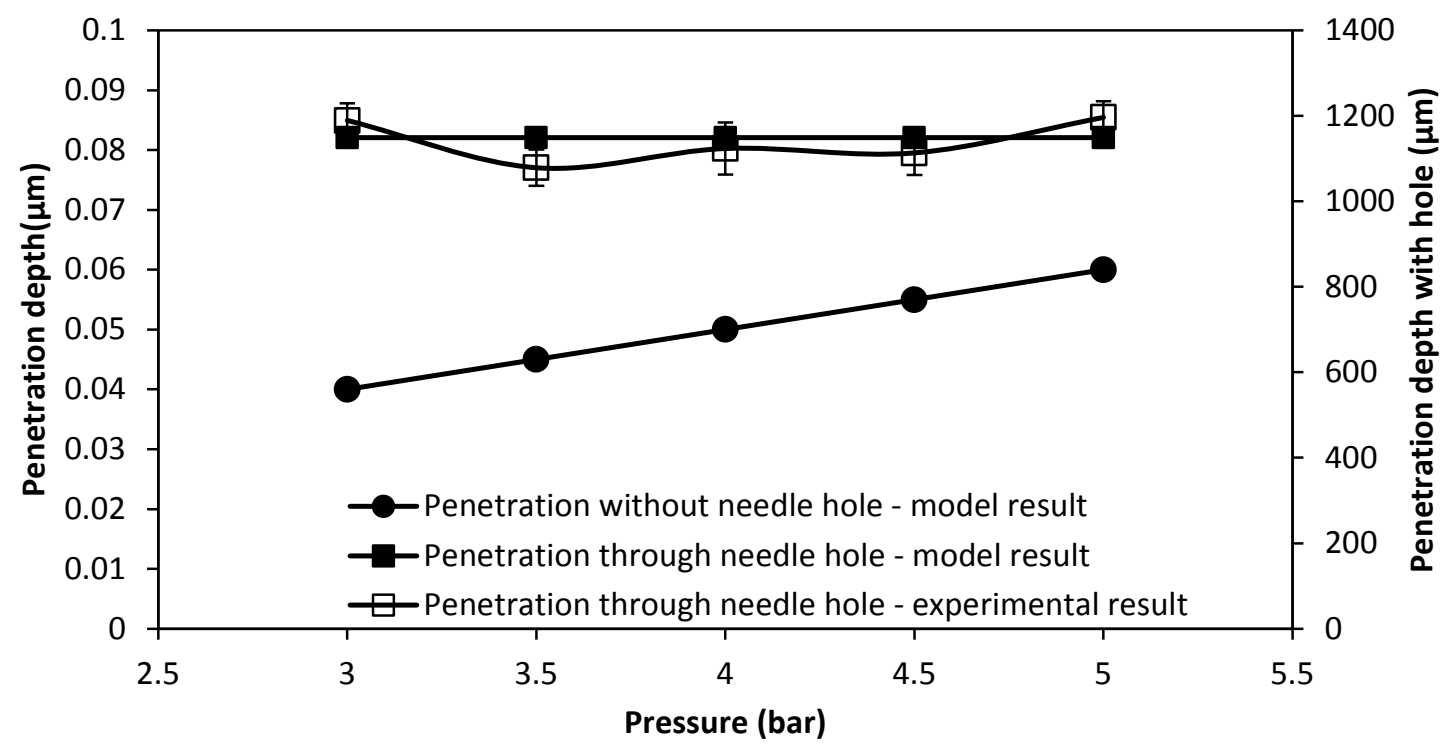

532

533

534

535

536

537

538

539

540

541

542

543

544

545

546

547

548

549

550

551

Figure 14: A comparison of penetration depth between model and experimental results (particle type: tungsten micro-particle of $3 \mu \mathrm{m}$ diameter). The experimental results in the figure are generated from three repeats of experiments.

A comparison of the penetration depth of the tungsten micro-particles $(3 \mu \mathrm{m})$ between model and experimental results is shown in Figure 14. It is worth to mention that the difference between experiment and modelling may be due to the assumption that the particle shape of the tungsten particle is regular after the separation stage in the model. As can be seen, the tungsten particles penetrate less than $0.1 \mu \mathrm{m}$ without using MN for the model result. It matches well with the experimental results which show the tungsten micro-particles cannot penetrate into the skin mimicking agarose gel. However, this condition can be made up by using MNs. As presented in Figure 14 the tungsten micro-particles reaches a further depth using Adminpatch MN 1500, but the maximum penetration depths are varied at pressure ranges from 3 to 5 bar. This is because it is not possible to ensure that the length of the pierced holes is constant each time using the same MN. As expected, the model results match well with the experimental results. In conclusion, the maximum penetration depth of tungsten micro-particles is directly related to the length of the pierced holes, and the operating pressure only presents a slight effect on the penetration depth in this case. To further understand the effect of pierced holes on the penetration depth of tungsten micro-particles, three different lengths of MNs (see Table 1) have used and discussed in the following section. 


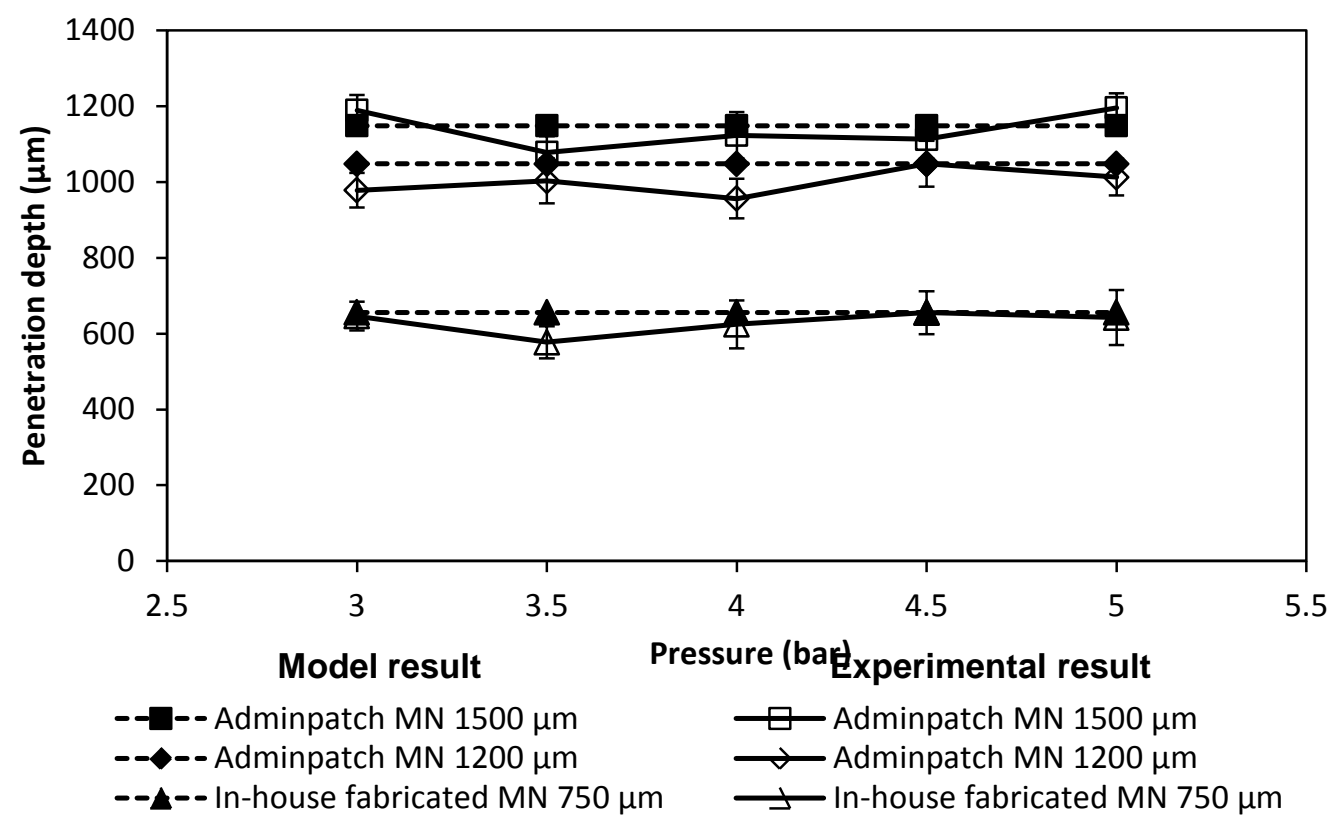

Figure 15: The effect of the micro-needle length on the maximum penetration depth of the tungsten particle (operating pressure: 5 bar; particle type: tungsten micro-particle of $3 \mu \mathrm{m}$ diameter). The experimental results in the figure are generated from three repeats of experiments.

\subsubsection{The effect of the micro-needle length}

In the real experiments, some tungsten micro-particles may be agglomerate after the separation stage. However, any effects of the particle agglomeration on the model results are not accounted for directly at this moment. This may be the reason why there are some differences between the experimental and modelling results; however, all comparison have produced reasonable match between the experimental and modelling results. Zhang et al. (2014) have shown that the length of pierced holes has a greater effect on the maximum penetration of stainless steel particles, which is related to the MN length. As expected, it agrees with the results of tungsten micro-particles shown in the Figure 15. As can be seen, the maximum penetration depth of tungsten micro-particles seems to be increased with an increase in the MN length. In principle, the length of pierced holes is directly related to the $\mathrm{MN}$ length which maximizes its length. An increased length of the pieced holes allows a number of micro-particles which deliver into the holes to penetrate further inside the target and maximizes the penetration depth. As expected, the experiment results are varied for each application of MNs due to difficulties maintaining a constant hole length. However, the model results match well with the experimental results, which illustrate the applicability of the model for MN assisted micro-particle delivery. 
the body of the same person (Xu et al., 2007; Vexler et al., 1999). As discussed earlier, the penetration depth of micro-particles is related to the yield force $\left(F_{y}\right)$, a frictional resistive force $\left(F_{f}\right)$ and a resistive inertial force of target material $\left(\mathrm{F}_{\mathrm{i}}\right)$ which decelerate the high-speed micro-particles inside the target (see equation 8). However, the yield force depends on the yield stress of the target (equation 11). Further, the resistive inertial force is related to the target viscosity (equation 9) and the frictional resistive force depends on the density of the target material (equation 10). In view of these inter-dependencies which in turn affect the micro-particle delivery, this section aims to analyse the effects of each force on the penetration depth of micro-particle to further investigate the major factors which provide a greater effect on the penetration depth.

For the $\mathrm{MN}$ assisted micro-particle delivery, the main point is the maximum penetration depth of the micro-particle in the skin. As discussed already, a number of the micro-particles penetrate through the holes made by the MNs to the dermis layer of the skin. In order to investigate the effect of each force on the maximum penetration depth of micro-particle, we assume that the micro-particles penetrate into the dermis layer at the tip area of the hole to obtain the maximum penetration depth of micro-particles. In addition, we kept one of those three resistive forces as constant and ignored the other two forces to analyse the variation of the penetration depth in the model. As shown in Figure 16, stainless steel micro-particles penetrated further than the tungsten particles due to the difference in particle size. Figure 16 also shows that the frictional resistive force provided a minimum effect on the micro-particle penetration. The effect of the yield force on the penetration depth is greater than the frictional resistive force. However, the major factor is the resistive inertial force which causes the penetration depth of micro-particles is almost negligible if the hole length (1149 $\mu \mathrm{m})$ is subtracted.

As discussed earlier, the resistive inertial force is a major component that determines the penetration depth of micro-particles for the gene gun based micro-particle delivery. Target viscosity directly affects the resistive inertial force. In order to further understand the effect of the resistive inertial force on the particle maximum penetration depth, the theoretical model is used to simulate the effect of varying the viscosity of the dermis layer from 5 to $19.6 \mathrm{MPa}$ where we kept the yield stress and density (see Table 2) and the hole length constant and operated the system at 5 bar operating pressure. As presented in Figure 17, the maximum penetration depth decreased from an increase in viscosity of the dermis layer. This is because an increased resistive inertial force slows down the particle velocity rapidly. Figure 17 also shows that the effect of a decreased viscosity of dermis layer on the maximum penetration depth is increased from an increase in 607 particle size and density due to an increased momentum. The maximum penetration depth of small 608 micro-particle (tungsten particle of $3 \mu \mathrm{m}$ diameter) is kept to approximately a constant when the viscosity of dermis layers is changed. 


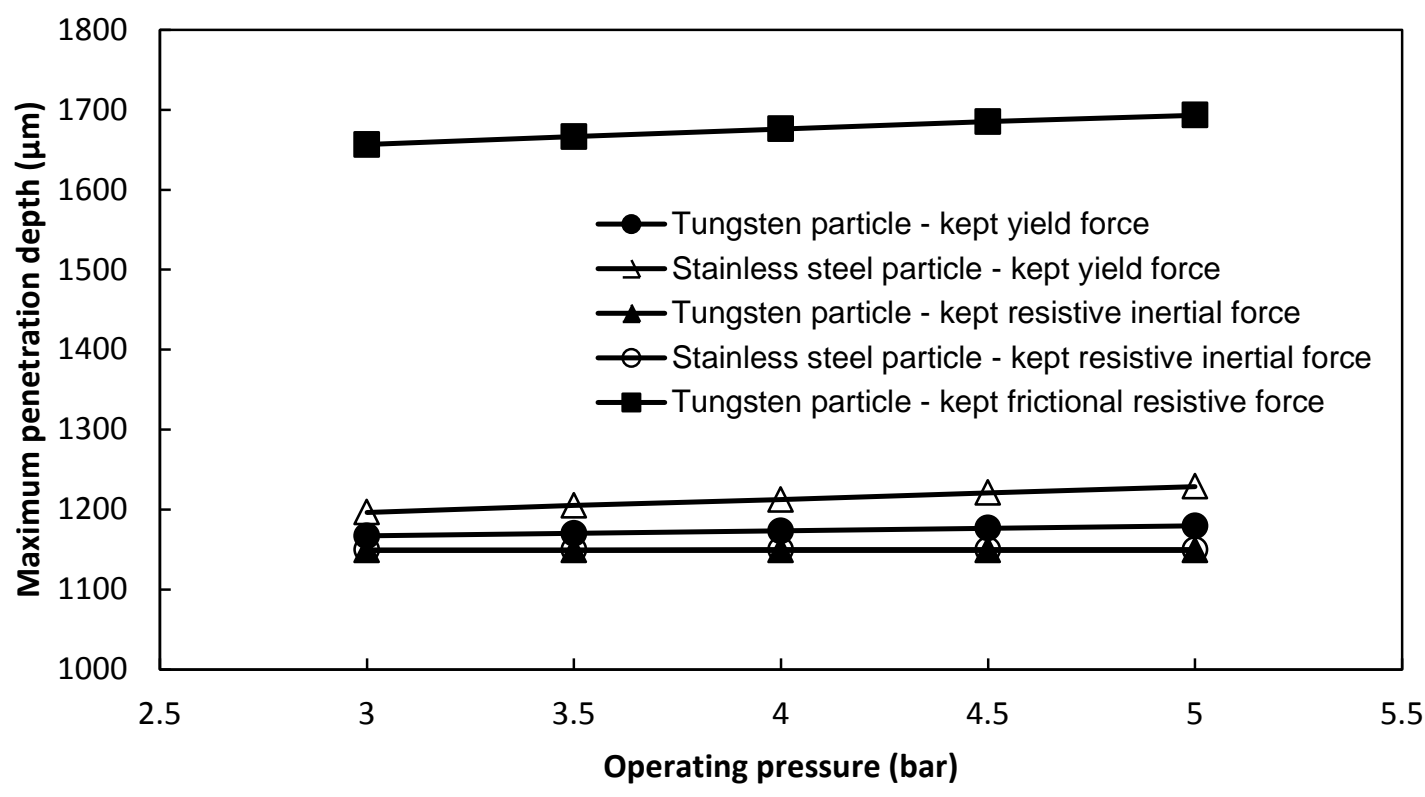

610

611 Figure 16: The effect of each resistive force on the maximum penetration depth of micro-particles (stainless steel particle: $18 \mu \mathrm{m}$ diameter; tungsten particle: $3 \mu \mathrm{m}$ diameter; Hole length: $1149 \mu \mathrm{m})$

\subsubsection{Further Discussions}

A comparison between stainless steel and tungsten micro-particles on the penetration depth in the skin mimicking agarose gel has been made in this study. Tungsten micro-particles are unable to penetrate the skin mimicking agarose gel/skin without using $\mathrm{MN}$ in the experimental result, which is different from the results of stainless steel micro-particles. The reason is that the diameter of tungsten micro-particles is less than $1 \mu \mathrm{m}$ which is too small when compared with the stainless steel micro-particles of 18 and $30 \mu m$ diameters. As a result the momentum of the tungsten micro-particles is too low to allow the particles to breach the gel surface. Zhang et al. (2013a) have measured the velocity of ground slide, which only reaches around $122 \mathrm{~m} / \mathrm{s}$ at 5 bar pressure, using a photoelectric sensor, which means that the particle velocity is less due to the energy loss. This velocity is less than some previous studies for gold particles, e.g., Kendal (2001) has shown that $1 \pm 0.2$ $\mu \mathrm{m}$ diameter gold particles can reach a velocity of $580 \pm 50 \mathrm{~m} / \mathrm{s}$ at 40 bar pressure using a contoured shock tube (CST). The ground slide presents a significant negative effect on the particle velocity, but it has safety advantage which prevents the pressurized gas from impacting the human body. Thus, the operating pressure is mimicked from 3 to 60 bar to study the penetration differences of tungsten micro-particles after increasing the momentum in the theoretical model. However, the tungsten micro-particles still cannot achieve the expected penetration depth. It indicates that a ground slide based gene gun system is not useful for the intercellular route because micro-particles cannot reach velocities high enough to breach the skin. Increasing the particle size will increase the moment but cause damage to the surface of the skin. As a result the extracellular route is the expected solution for normally ground slide based gene gun system. 


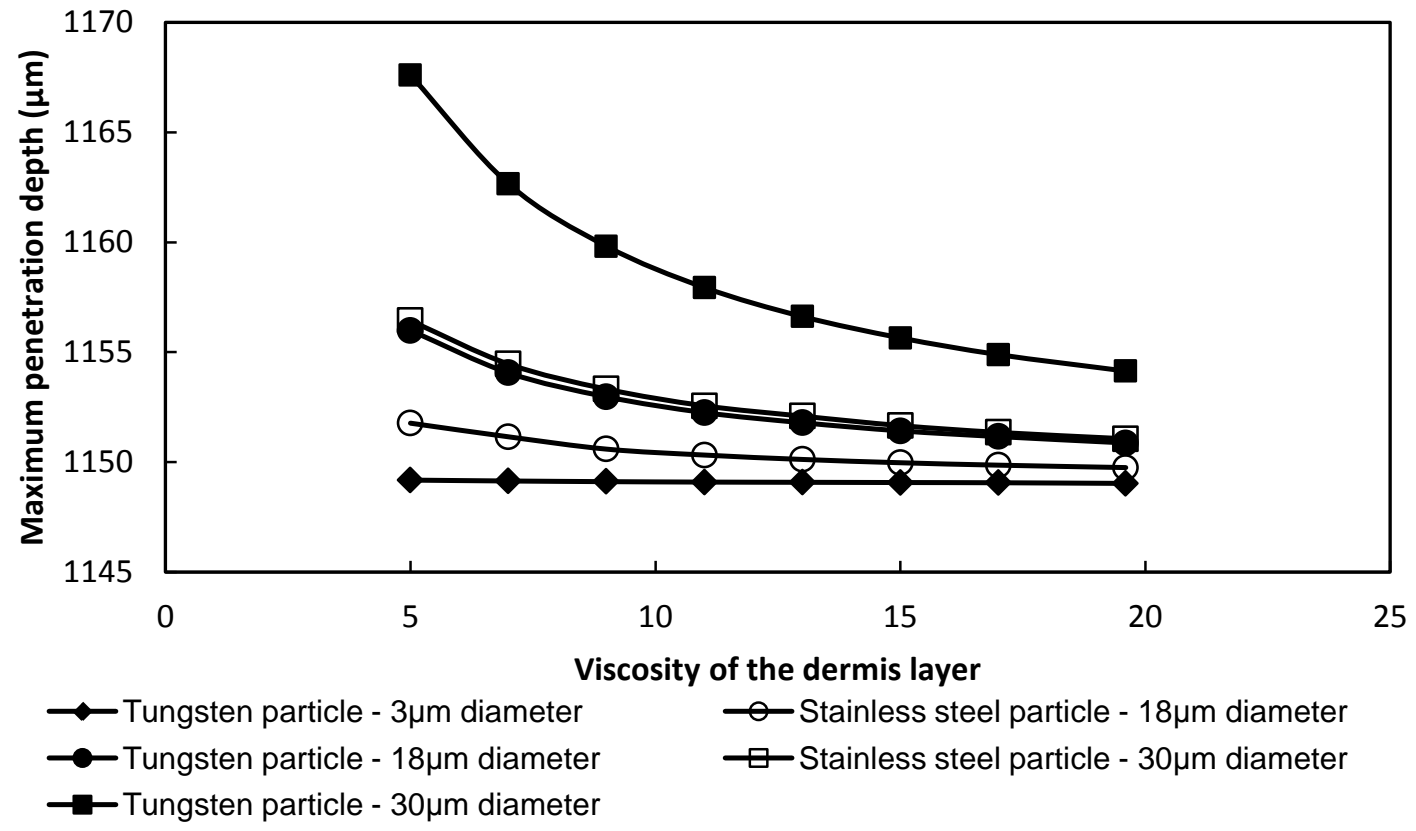

635 Figure 17: The effect of the viscosity of dermis layer on the maximum penetration depth of micro-particles 636 (operating pressure: 5bar; hole length: $1149 \mu \mathrm{m}$ )

However, the use of MN meets the purpose of an intercellular route to deliver micro-particle to a greater penetration depth in the target using a ground slide based gene gun system. Previously, Zhang et al. (2013a) have shown that the penetration depth of stainless steel micro-particles is increased while using a MN. In this paper, the tungsten micro-particles are investigated using modelling and experiments with MN assisted micro-particle delivery. Both model and experimental results show that the use of MN increases the penetration depth of tungsten micro-particles. However, the maximum penetration depth of micro-particles depends on the length of pierced holes which is created by MNs. The maximum penetration of tungsten micro-particles reaches around $1149 \mu \mathrm{m}$ using Adminpatch 1500 MM, which has possibly never reached for other relevant gene gun systems. For example, Kendal (2001) has indicated that gold particles of $1 \pm 0.2 \mu \mathrm{m}$ diameters can penetrate $66 \mu \mathrm{m}$ in the skin at velocity of $580 \pm 50 \mathrm{~m} / \mathrm{s}$ using a contoured shock tube (CST). Kendal et al. (2004) have also used a convergent-divergent device to accelerate $1.8 \mu \mathrm{m}$ diameter gold particles which achieves a maximum penetration depth of $78.6 \mu \mathrm{m}$ at 60 bar. Mitchell et al. (2003) has shown that gold particles of 1-3 $\mu \mathrm{m}$ diameters can reach a maximum depth of around $60 \mu \mathrm{m}$ in canine tissue at a velocity of $550 \pm 50 \mathrm{~m} / \mathrm{s}$. It is concluded that the diameters of gold particles ranges from 1 to 3 only can penetrates to a depth from 60 to $78.6 \mu \mathrm{m}$ (epidermis layer) which is less than the maximum penetration depth of the tungsten particles after using MNs. It shows that the improvement of the penetration depth using MN assisted micro-particle delivery, which achieves depths never reached before. In conclusion, the results show that the intercellular route is feasible for the MN assisted micro-particle delivery. In theory, it is the preferred route for MN assisted micro-particle delivery due to the cell damage is minimized from a decrease in particle size (O’Brien et al., 2011), although extracellular route presents more penetration depth. It could be proved by firing the above stainless steel/tungsten micro-particles into cell cultured skin mimicking agarose gel to 
analyse the cell damage in further work. Future work should also focus on using animal/human skin for detailed histological studies in contrast to agarose gel as used in this study. If using the proposed methods, one should also be aware of the likeliness of dermal damage and pain that may occur if using long microneedles besides any effect of gene gun on mechanical damage of the skin. Therefore, safety studies should be conducted in detail in future studies.

\section{Conclusions}

This study shows an effective method for relating the use of MNs to create holes on the target for micro-particle delivery to improve the penetration depth of micro-particles. The experimental rig considers possible changes in the operating pressure to analyse the penetration depth and passage percentage of tungsten and stainless steel micro-particles. Results show that the passage percentage increases due to an increase in operating pressure until a maximum value. Tungsten micro-particles used in this study present a lower passage percentage than stainless steel due to an insufficient momentum to reach the target (particle collector). In addition, tungsten micro-particles are stopped by the skin mimicking agarose gel even if the operating pressure is increased. But an increased operating pressure improves the penetration of stainless steel particle without using MNs. This is because the diameter of tungsten micro-particles is small $(<1 \mu \mathrm{m})$. To further understand the particle size and density effect on the penetration depth, a theoretical model is used to mimic the operating pressure ranges from 3 to 60 bar to accelerate tungsten and stainless steel micro-particles while the diameter ranges from 3 to $100 \mu \mathrm{m}$. The results show a great improvement in the penetration depth of tungsten micro-particles which is more than stainless steel micro-particles. However, both model and experimental results show that a use of MNs provides an improvement on the penetration although the momentum is insufficient to breach the skin. The maximum penetration depth of micro-particles up to dermis layer has not been reached before. It is related to length of MNs which determine the length of pierced holes. As expected, model results matches well with the experimental results, which illustrates the applicability of the model for the MN assisted micro-particle delivery. MN assisted micro-particle delivery improves safety because an intercellular route can be chosen to reduce the damage from particle impact and the pressurized gas is prevented by the ground slide. In addition, it also enhances the penetration depth of micro-particles properly if compared with any other gene gun systems. An increased penetration depth allows deeper tissue to be transfected, which provides more effective gene transfection in the target. Future work could be to attach the genes on the micro-particles, fired into the cells using MN assisted micro-particle delivery and then analyse the DNA profile of the cells to verify the advantage of MN assisted micro-particle delivery.

\section{Acknowledgement}

Loughborough University (UK) is acknowledged for providing a PhD studentship to Dongwei Zhang which made this work possible. Furthermore, the technical supports from Mr Tony Eyre, Mr Mark Barron, Mr Jim 


\section{Conflict of Interest}

698

The authors declare no conflict of interest

699

\section{References}

Al-Qallaf B., Das D.B., Davidson A., (2009). Transdermal Drug Delivery by Coated Microneedles: Geometry Effects on Drug Concentration in Blood, Asia-Pacific Journal of Chemical Engineering, 4(6): 845-857, DOI: 10.1002/apj.353.

Arora A., Prausnitz M.R., Mitragotri S., (2008) Micro-scale devices for transdermal drug delivery, Int. J. Pharm.

Bastian S., Busch W., Kuhnel D., Springer A., Holke R., Scholz S., Pompe W., Gelinsky M., Potthoff A., Richter V., Ikonomidou C., Schirmer K., (2009). Toxicity of tungsten carbide and cobalt-doped tungsten carbide nanoparticles in mammalian cells in vitro, Environ. Health Perspect,117(4): 530-536

Bennett A.M., Phillpotts R.J., Perkins S.D., Jacobs S.C., Williamson E.D., (1999). Gene gun mediated vaccination is superior to manual delivery for immunisation with DNA vaccines expressing protective antigens from Yersinia pestis or Venezuelan Equine Encephalitis virus, Vaccine, 18(7-8): 588-596

Crozier W.D., Hume W., (1957). High velocity light gas gun, Journal of Applied Physics, 28(8): 892-895

Cormier M., Johnson B., Ameri M., Nyam K., Libiran L., Zhang D. D., and Daddona P.. (2004). Transdermal delivery of desmopressin using a coated microneedle array patch system. J. Control. Release, 97: 503-511

Davis S.P., Landis B.J., Adams Z.H., Allen M.G., Prausnitz M.R.. (2004). Insertion of microneedles into skin:

Dehn J. (1987). A Unified theory if penetration, International journal of impact engineering, 5: 239-248 
Donnelly R.F., Garland M.J., Singh T.R.R., Migalska K., Majithiya R., McCrudden C.M., Kole P.L., Mahmood T.M.T., McCarthy H.O., Willfson A.D. (2012). Hydrogel-Forming microneedle array for enhanced transdermal drug delivery, Adv. Funct. Mater., 22:4879-4890.

Vexler A., Polyansky I., Gorodetsky R., (1999). Evaluation of skin viscoelasticity and anisotropy by measurement of speed of shear wave propagation with viscoelasticity skin analyser, Journal of Investigative Dermatology, 113: 732-739

Giudice E.L., Campbell J.D., (2006). Needle-free vaccine delivery, Adv.Drug Deliv. Rev. 58: 68-89

Han T., Das D.B. (2013). Permeability enhancement for transdermal delivery of large molecule using low-frequency sonophoresis combined with microneedles, Journal of Pharmaceutical Science, 102(10): 3614-3622

Hardy M.P., Kendall M.A.F., (2005). Mucosal deformation from an impinging transonic gas jet and the ballistic impact of micro-particles, Phys. Med. Biol., 50: 4567-4580

Holbrook, K. A., Odland, G.F. (1974). Regional Differences in the thickness (cell layers) of the human stratum corneum: an ultrasound analysis, J Invest Dermatol., 62: 415-422.

Kalluri H., Banga A.K.. (2011). Formation and closure of microchannels in skin following microporation, Pharm. Res., 28: 82-94

Kendall M.A.F., Carter F.V., Mitchell T.J., and Bellhouse B.J., (2001). Comparison of the transdermal ballistic delivery of micro-particle into human and porcine skin, Proceedings of the 23rd Annual International Conference of the IEEE: 2991-94

Kendall M.A.F., Rishworth S., Carter F., Mitchell T., (2004). Effects of relative humidity and ambient temperature on the ballistic delivery of micro-particles to excised porcine skin, J. Invest. Dermatol. 122: 739-746.

Kis E.E., Gerhard W., Myschik J., (2012). Devices for intradermal vaccination, Vaccine, 30: 523-538

Kishino A., Yanagida T., (1988). Force measurements by micromanipulation of a single actin filament by glass needles, Nature, 334: 74-76 
Klein T.M., Wolf E.D., Wu R. and Sanford J.C., (1987). High-velocity microprojectiles for delivering nucleic acids into living cells, Nature, 327: 70-73

Liu Y., (2006). Physical-mathematical modelling of fluid and particle transportation for DNA vaccination, International Journal of Engineering Science, 44: 1037-1049

Liu Y., (2007). Impact studies of high-speed micro-particle following biolistic delivery, IEEE transactions of biomedical engineering, Transactions on Biomedical Engineering, 54(8): 1507-1513

Macklin, M.D., Drape, R.J., Swain, W.F., (2000). "Preparation for particle-mediated gene transfer using the Accell gene gun", Methods in molecular medicine, 29: 297-303

Matteucci, M., Fanetti, M., Casella, M., Gramatica, F., Gavioli, L., Tormen, M., Grenci, G., De Angelis, F., Di Fabrizio, E. (2009) Poly vinyl alcohol re-usable masters for MN replication. Microelectronic Engineering, 86(4-6): 752-756 doi:10.1016/j.mee.2009.01.068

McAllister D.V., Wang P.M., Davis S.P., Park J.H., Canatella P.J., Allen M.G., Prausnitz M.R.. (2003). Microfabricated needles for transdermal delivery of macromolecules and nanoparticles: fabrication methods and transport studies. PNAS, 100: 13755-13760

Menezes V., Mathew Y., Takayama K., Kanno A., Hosseini H., (2012). Laser plasma jet driven microparticles for DNA/drug delivery, PLOS ONE, 7(11): e50823

Mitchell T.J., Kendall M.A.F., Bellhouse B.J., (2003). A ballistic study of micro-particle penetration to the oral mucosa, International Journal of Impact Engineering, 28: 581-99

Miyano T., Tobinaga Y., Kanno T., Matsuzaki Y., Takeda H., Wakui M., and Hanada K., (2005). Sugar micro needles as transdermic drug delivery system. Biomed. Microdevices, 7: 185-188

Nayak A., Das D.B. (2013). Potential of biodegradable microneedles as a transdermal delivery vehicle for lidocaine, Biotechnology Letters, 35(9): 1351-1363

Nayak A., Das D.B., Vladisavljević, GT (2013). Microneedle-Assisted Permeation of Lidocaine Carboxymethylcellulose with Gelatine Co-polymer Hydrogel. Pharmaceutical Research, DOI: $10.1007 / s 11095-013-1240-z$ (in press) 
O'Brien J.A., Lummis S.C.R., (2011). Nano-biolistics: a method of biolistic transfection of cells and tissues using a gene gun with novel nanometer-sized projectiles, BMC Biotechnology, 11; 66

Olatunji, O., Das, D.B. (2010). Painless Drug delivery using microneedles. Current technologies to increase the transdermal delivery of drugs (Editor: Joan Escober Chavez). Bentham Science Publishers (available online at http://www.benthamdirect.org/pages/b_getarticlebybook.php). ISBN:978-1-60805-191-5.

Olatunji, O., Das, D.B. (2011). Drug delivery using Microneedles. Comprehensive Biotechnology (Editor: Zhanfeng Cui). 2nd Edition, MS number 501. MRW, Elsevier, The Boulevard, Langford Lane, Oxford, United Kingdom. ISBN: 13: 978-0-444-53352-4

Olatunji, O, Das, DB, Nassehi, V (2012) Modelling transdermal drug delivery using microneedles: Effect of geometry on drug transport behaviour, Journal of Pharmaceutical Sciences, 101(1): 164-175, ISSN: 0022-3549. DOI: 10.1002/jps.22736.

Olatunji O., Das D.B., Garland M.J., Belaid L., Donnelly R.F., (2013). Influence of Array interspacing on the force required for successful microneedle skin penetration: theoretical and practical approaches, Journal of Pharmaceutical Science, 102(4): 1209-1221.

Quinlan N.J., Kendall M.A.F., Bellhouse B.J., Ainsworth R.W., (2001). Investigations of gas and particle dynamics in first generation needle-free drug delivery device, Shock Waves, 10: 395-404

Ritman E.L., (2004). Micro-computed tomography - Current status and developments, Annual review of Biomedical Engineering, 6: 185-208

Russell J.A., Roy M.K., Sanford J.C., (1992). Physical trauma and tungsten toxicity reduce the efficiency of biolistic transformation, Plant Physiol., 98: 1050-1056

Sato H., Hattori S., Kawamoto S., Kudoh I., Hayashi A., Yamamoto I., Yoshinari M., Minami M., Kanno H., (2000). In vivo gene gun-mediated DNA delivery into rodent brain tissue, Biochem. Biophys. Res. Commun., 270(1): 163-170.

Schaefer, H., Redelmeier, T.E. (1996) Skin barrier: Principles of percutaneous absorption, New York, Karger. Soliman S.M. (2011). Micro-particle and Gas Dynamics in an Axi-symmetric Supersonic Nozzle, University of Cincinnati (Cincinnati, USA), Thesis for the degree of Doctor of Philosophy in Aerospace Engineering. 
Soliman S.M., Abdallah S., (2011). CFD investigation of powdered vaccine and gas dynamics in biolistic gun,

Soliman S.M., Abdallah S., Gutmark E., Turner M.G., (2011). Numerical simulation of microparticles penetration and gas dynamics in an axi-symmertric supersonic nozzle for genetic vaccination, Powder

Thomas J.L., Bardou J., Mauchamp B. et al, Chavancy Gerard (2001). A helium burst biolistic device adapted to penetrate fragile insect tissues, Journal of Insect Science, 1(9): 1-10

Trainer A.H., Alexander M.Y., (1997). Gene delivery to the epidermis, Human molecular genetics, 6(10): 1761-1767

851

Uchida M., Li X.W., Mertens P., Alpar H.O., (2009). Transfection by particle bombardment: Delivery of plasmid DNA into mammalian cells using gene gun, Biochim. Biophys. Acta., 1790(8):754-764

Walters K.A., Roberts M.S., (2007). Dermatologic, Cosmeceutic, and Cosmetic Development, Therapeutic and Novel Approaches, CRC press, Chapter 36: 591-611

Webster C., (1995). The Discovery of Boyle's law, and the concept of the Elasticity of Air in the Seventeenth 860

Wildnauer R.H., Bothwell J.W., Douglas A.B., (1971). Stratum corneum properties I. Influence of relative humidity on normal and extracted stratum corneum, J. Invest. Dermatol., 56: 72-78

Williams R.S., Johnston S.A., Riedy M., DeVit M.J., McElligott S.G., Sanford J.C., (1991). Introduction of foreign genes into tissues of living mice by DNA-coated microprojectiles, Proc. Natl. Acad. Sci. U.S.A., 88:

Xu F., Wen T., Seffen K.A., LuT.J., (2007). Characterization of Thermomechanical behaviour of skin tissue Viscoelastic behaviour, Proceedings of the World Congress on Engineering, 2: WCE 2007

Yoshida Y., Kobayashi E., Endo H., Hamamoto T., Yamanaka T., Fujimura A., Kagawa Y., (1997). Introduction of DNA into rat liver with a hand-held gene gun: Distribution of the expressed enzyme, [32P] DNA, and Ca2+ flux, Biochem. Biophys. Res. Commun. 234(3): 695 -700. 
874

875 Yoshimisu Y., Tanaka K., Tagawa T., Nakamura Y., Matsuo T., Okamoto S., (2009). Improvement of 876 DNA/Metal Particle Adsorption in Tungsten-Based Biolistic Bombardment; Alkaline pH is Necessary for DNA 877 Adsorption and Suppression of DNA Degradation, Journal of Plant Biology, 52: 524-532.

878

879 Zhang D.W., Das D.B., Rielly C.D., (2013a). An experimental study of microneedles assisted micro-particle 880 delivery using a model system, Journal of Pharmaceutical Science, 102(10): 3632-3644, DOI: $881 \quad 10.1002 / j p s .23665$.

882

883 Zhang D.W., Das D.B., Rielly C.D., (2013b). Potential of microneedles assisted micro-particles delivery from 884 gene gun: A review. Drug Delivery, DOI: 10.3109/10717544.2013.864345 (in press).

885

886 Zhang D.W., Das D.B., Rielly C.D., (2014). Microneedles assisted micro-particles delivery from gene guns:

887 Experiments using skin mimicking agarose gel. Journal of Pharmaceutical Sciences, DOI: 10.1002/jps.23835 888 (in press). 